\title{
Pseudomonas aeruginosa modulates alginate biosynthesis and type VI secretion system in two critically ill COVID-19 patients
}

\author{
Jiuxin $\mathrm{Qu}^{1 \dagger}$, Zhao $\mathrm{Cai}^{2+}$, Xiangke Duan ${ }^{2}$, Han Zhang ${ }^{2}$, Hang Cheng ${ }^{2}$, Shuhong Han ${ }^{2}$, Kaiwei Yu ${ }^{2}$, \\ Zhaofang Jiang ${ }^{1}$, Yingdan Zhang ${ }^{2}$, Yang Liu ${ }^{3}$, Fang Bai ${ }^{4}$, Yingxia Liu ${ }^{1,5}$, Lei Liu ${ }^{1,5^{*}+}$ and Liang Yang ${ }^{1,2,6^{*}+}$
}

\begin{abstract}
Background: COVID-19 pneumonia has caused huge impact on the health of infected patients and associated with high morbidity and mortality. Shift in the lung microbial ecology upon such viral infection often worsens the disease and increases host susceptibility to superinfections. Bacterial superinfection contributes to the aggravation of COVID-19 and poses a great challenge to clinical treatments. An in-depth investigation on superinfecting bacteria in COVID-19 patients might facilitate understanding of lung microenvironment post virus infections and superinfection mechanism.
\end{abstract}

Results: We analyzed the adaptation of two pairs of P. aeruginosa strains with the same MLST type isolated from two critical COVID-19 patients by combining sequencing analysis and phenotypic assays. Both $P$. aeruginosa strains were found to turn on alginate biosynthesis and attenuate type VI secretion system (T6SS) during short-term colonization in the COVID-19 patients, which results in excessive biofilm formation and virulence reduction-two distinct markers for chronic infections. The macrophage cytotoxicity test and intracellular reactive oxygen species measurement confirmed that the adapted $P$. aeruginosa strains reduced their virulence towards host cells and are better to escape from host immune clearance than their ancestors.

Conclusion: Our study suggests that SARS-CoV-2 infection can create a lung environment that allow rapid adaptive evolution of bacterial pathogens with genetic traits suitable for chronic infections.

Keywords: Pseudomonas aeruginosa, COVID-19, Bacterial superinfection, Type VI Secretion System, Biofilm

*Correspondence: Liulei3322@aliyun.com; yangl@sustech.edu.cn ${ }^{\dagger}$ Jiuxin Qu and Zhao Cai contributed equally to this work

†Lei Liu and Liang Yang contributed equally to this work and share the corresponding authorship

1 Department of Clinical Laboratory, Shenzhen Third People's

Hospital, Second Hospital Affiliated to Southern University of Science and Technology, Guangdong Provincial Clinical Research Center

for Infectious Diseases (Tuberculosis), National Clinical Research Center for Infectious Diseases, Shenzhen 518000, Guangdong, China

${ }^{2}$ School of Medicine, Southern University of Science and Technology, Shenzhen 518055, China

Full list of author information is available at the end of the article

\section{Background}

Coronavirus Disease 2019 (COVID-19) induced by Severe Acute Respiratory Syndrome Coronavirus 2 (SARS-CoV-2) has caused over 4.7 million death globally as reported by WHO at Sep 2021. SARS-CoV-2 induces cytokine storm and hyperinflammation resulting in respiratory dysfunction and necrosis of epithelial cells of the lungs $[1,2]$. Such histological changes in the lung tissues may result in dysbiosis of lung microbiome and give rise to secondary bacterial infection in COVID-19 patients [3]. Several groups of researchers have emphasized that microbial superinfection indeed occurs in COVID-19 patients, especially in severe cases, exacerbating disease 
progress and making difficulties to clinical treatments [4-7]. Nosocomial bacterial pathogens are risk factors contributing to virus superinfections which can lead to increased death of critically ill COVID-19 patients $[8,9]$. The common superinfecting bacteria reported include Mycoplasma pneumonia, Staphylococcus aureus, Klebsiella spp., Acinetobacter baumannii, Haemophilus influenzae, Pseudomonas aeruginosa and etc. [10]. Bacteremia cases caused by Pseudomonas were also being reported [11].

P. aeruginosa is identified as a top superinfecting bacteria with SARS-CoV-2 in the Second Affiliated Hospital of Southern University of Science and Technology, China [12]. P. aeruginosa is a notorious nosocomial infection pathogen causing fatal infections in immunocompromised patients with diseases like cystic fibrosis, ventilator-associated pneumonia (VAP), catheter-associated infections and burn wounds [13, 14]. Patients with severe COVID-19 requiring ECMO had a very high rate of late-onset VAP, which is frequently caused by $P$. aeruginosa, with multiple recurrences and difficulties eradicating the pathogen from the lung [15]. This bacterium causes infections through different mechanisms, including secretion of a variety of virulence factors and biofilm formation [13]. P. aeruginosa engages diverse virulence mechanisms including quorum sensing systems, protein secretion systems, exopolysaccharides etc. for competition in the polymicrobial environments and escaping the host immune attack [13]. Longitudinal analysis of convergent evolution of $P$. aeruginosa clinical isolates from chronic lung infections showed that remodeling of biofilm formation and virulence factor is essential for host adaptation [16, 17]. Mutations in genes like $m u c A, v g r G$, lasR, rpoN and $p v d S$ significantly impair quorum sensing systems, protein secretion systems and biosynthesis of virulence factors, and allow $P$. aeruginosa to escape from host immune clearance in patients with lung diseases such as cystic fibrosis and ventilator-associated pneumonia [16-19]. Among the protein secretion systems, the type VI secretion system (T6SS) is particularly important for $P$. aeruginosa to compete with other microbial species and impair host cells [20]. Analysis of these evolutionary traits could contribute to the development of therapeutic measures to treat infections caused by $P$. aeruginosa.

Currently, characterization of bacterial adaptive evolution in COVID-19 patients is rare, which might limit our understanding of the lung microenvironment post SARS-CoV-2 infection. Although a recent study reported the change of $P$. aeruginosa in critically ill COVID-9 patients, it mainly focused on the evolution of its antimicrobial susceptibility over time [21]. To address this, we sequenced and compared the genomes and transcriptomes of four $P$. aeruginosa isolates sampled longitudinally from two critically ill COVID-19 patients, two isolates from each patient, to investigate its adaptation and pathogenesis patterns. The isolates collected from different patients are of the same sequence typing (ST type), indicating the possible occurrence of hospitalacquired $P$. aeruginosa infection. Interestingly, the later $P$. aeruginosa isolates form excessive biofilm by increasing alginate biosynthesis and developing mucoid phenotype, which are usually selected after long-term colonization in the lung environment. More notably, we demonstrated that $P$. aeruginosa attenuates its T6SS, especially HSI-II T6SS, to suppress its virulence and escape host clearance during superinfection. Our study suggests that SARSCoV-2 infection can create a lung environment that allow rapid adaptive evolution of bacterial pathogens with genetic traits suitable for chronic infections.

\section{Results}

Post viral $P$. aeruginosa infection in the respiratory track of COVID-19 patients

During routine screening of respiratory samples (sputum samples and bronchioalveolar lavage fluids) of COVID19 patients, we discovered that two patients were colonized by $P$. aeruginosa. These two patients were living in the same ward in the department of Infectious Diseases. Clinical data of the two patients indicated that they were diagnosed to have bacterial pneumonia in addition to COVID-19 pneumonia. In total, four $P$. aeruginosa isolates were collected from respiratory samples, sputum samples or bronchioalveolar lavage fluids (BALF) samples of the two patients and named as LYSZa2, LYSZa3, LYSZa5 and LYSZa6 respectively. Among which, LYSZa2 (sputum) and LYSZa3 (BALF) were isolated from patient 1 on day 12 (the day of ventilation) and day 15 of hospitalization, while LYSZa5 (BALF) and LYSZa6 (sputum) were isolated from patient 2 on day 17 (10 days after ventilation) and day 32 of hospitalization. We defined LYSZa2 and LYSZa5 as the ancestry isolates while LYSZa3 and LYSZa6 as the progeny isolates. To investigate epidemiological link between these $P$. aeruginosa isolates, genomes of all isolates were sequences by using Illumina HiSeq platform. Multi-Locus Sequence Typing (MLST) analysis indicated that four isolates collected from these two patients are of the same type, $P$. aeruginosa ST1074, suggesting that these $P$. aeruginosa isolates could be hospital-acquired. To find out if $P$. aeruginosa is the dominant superinfecting species in these COVID-19 patients causing post-viral bacterial infection, we assessed the microbiome of the sputum samples using metagenomics sequencing analysis. Results of taxonomic classification and species abundance analysis indicated that $P$. aeruginosa is the most abundant species in the microbiome of all four sputum samples (Additional file 1: Fig. S1A and 
B). Alpha diversity analysis indicated that there is no significant difference in the composition of microbiome between the progeny isolates and the ancestor isolates (Additional file 1: Fig. S1C). Since P. aeruginosa is well known to engage adaptive evolution during colonization in the respiratory track $[16,17]$, we then focused on these isolates to analyze their adaptive evolutionary traits during colonization in COVID-19 environment. Genomes of the first isolate of each patient, LYSZa2 and LYSZa5, were further sequenced using Pacific Biosciences RSII sequencer for further analysis.

\section{Characterization of genomes, phylogeny and antibiotic resistance of $P$. aeruginosa isolates}

Circular genomes of LYSZa2 and LYSZa5 were assembled while the genome sizes are 6, 638, 980 bp and 6, 638, 990 bp, with $66.2 \%$ of GC respectively. Rearrangements were checked and manually curated after pairwise comparison of the two genomes. As predicted by PGAP, LYSZa2 genome contains 6183 genes among which 6046 features encode for proteins and 86 encode for RNAs, while LYSZa5 genome contains 6183 genes among which 6045 features encode for proteins and 86 encodes for RNAs. These two genomes are almost identical, which again suggests a hospital acquired infection and indicates that they are of same clone.

Both of the $P$. aeruginosa genomes contain the same antimicrobial resistance genes predicted by ResFinder, including crpP, aph(3')-IIb, catB7, blaOXA-50, blaPAO, and $f o s A$ against fluoroquinolone, aminoglycoside, phenicol, beta-lactam and fosfomycin drugs. No difference in antimicrobial genes was identified between the ancestry and the progeny isolates. According to the clinical information, patient 1 (LYSZa2\&3) received a series of antimicrobials until the progeny $P$. aeruginosa was isolated, including moxifloxacin or piperacillin-tazobactam, or meropenem; while patient 2 (LYSZa5\&6) received a series of antimicrobials until the progeny $P$. aeruginosa was isolated, including moxifloxacin or cefoperazonesulbactam, or meropenem combined with vancomycin. In vitro antimicrobial resistance of the isolates was tested using antibiotics including ceftazidime, piperacillin, cefoperazone/sulbactam, imipenem, aztreonam and levofloxacin according to the antibiotic susceptibility guidelines provided by CLSI. Obvious differences in resistance were observed between the ancestry and the progeny isolates. LYSZa5 is sensitive to ceftazidime, piperacillin, cefoperazone/sulbactam, imipenem and aztreonam while LYSZa6 becomes intermediate or resistance to these antibiotics after 15 days of evolution probably due to the upregulation of mexAB-oprM genes encoding for efflux pump (Additional file 3: Table S1). This increase in the expression of these efflux pump genes was probably due to the downregulation of their transcriptional regulator, nalC gene (Additional file 9: Table S7). Antibiotic treatment is probably a driver for such evolution. However, no obvious change in resistance to these drugs was observed between LYSZa2 and LYSZa3 probably due to the short evolving time (Additional file 3: Table S1).

Genomic islands (GIs) on LYSZa2 and LYSZa5 genomes were predicted by IslandViewer4 (Additional file 4: Table S2 Additional file 5: TableS3). In total, 35 GIs on LYSZa2 and 36 GIs on LYSZa5 were predicted by at least one prediction method. Genomes of LYSZa2 and LYSZa5 were compared with genomes of five other $P$. aeruginosa strains including PAO1 reference strain and four virulence strains, PA14, LESB58, SCV20265 and VFRPA04 (Fig. 1A). Most of the GIs predicted are specific to LYSZa2 and LYSZa5 genomes (Fig. 1A). Genes in these GIs are involved in transcriptional regulation, DNA restriction-modification, DNA repair, toxin-antitoxin and secretion systems, showing that these GIs maybe important for $P$. aeruginosa survival and virulence during superinfection with SARS-CoV-2.

We then traced the origin of these isolates by constructing phylogenetic tree using core genomes of LYSZa2 and LYSZa5 with 22 other clinical or environmental $P$. aeruginosa genomes selected from NCBI/Pseudomonas genome database (Additional file 6: Table S4) and one SARS-CoV-2 superinfecting strain published by our group recently, $P$. aeruginosa LYSZa7[22]. As seen from the phylogenetic tree (Fig. 1B), LYSZa2 and LYSZa5 are closed related without evolutionary distance between them, and are phylogenetically approximate to the PAO1 reference strain and the hypervirulent isolate LESB58 from a CF patient, rather than the hypervirulent PA14 strain.

Single Nucleotide Polymorphism (SNP) and other genome modifying events were assessed between the ancestry isolates and the progeny isolates respectively using PAO1 as reference (Additional file 7: Table S5). 90 genomic modifying events including nucleotide variation, insertion, deletion and replacement (Additional file 7: Table S5) were identified in LYSZa3 as compared to LYSZa2. 93 of such genomic modifying events (Additional file 7: Table S5) were identified in LYSZa6 as compared to LYSZa5. Common mutations were found between LYSZa3 and LYSZa6 on genes related to type VI secretion system and iron transport when compared with their ancestors, respectively (Table 1). Such observation indicated that $P$. aeruginosa undergoes adaptive evolution in COVID-19 patients to survive and modulate its virulence during the post virus infection. We then performed RNA sequencing to evaluate the changes at transcriptional level during $P$. aeruginosa evolution. 


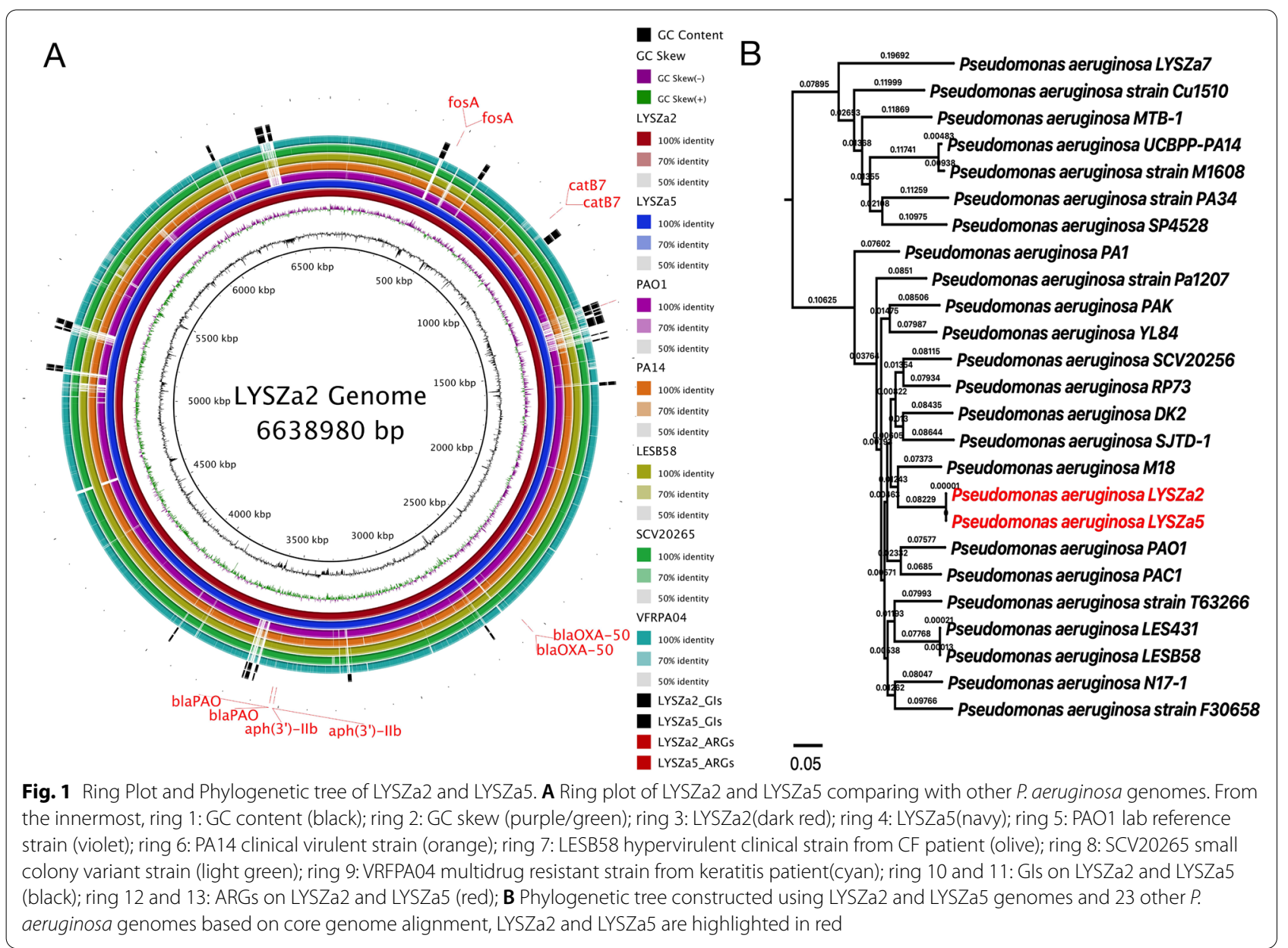

Increase in alginate biosynthesis of $P$. aeruginosa during adaptation in COVID-19 patients

To further elucidate the $P$. aeruginosa adaptation during colonization in the COVID-19 patients, we performed RNA sequencing analysis of these isolates using Illumina HiSeq platform and focused on discovering the differential expressed genes between the ancestry and progeny isolates longitudinally. Moreover, we also compared the differential gene expression between the progeny cells of the two patients to learn the parallel changes of $P$. aeruginosa in different hosts. Based on the filtering criteria of fold change $\geq 4$, adjusted $p$-value $<0.05$ and base mean $\geq 20$, the expression of 129 genes were differentially regulated in LYSZa3 as compared to LYSZa2, among which 75 were upregulated and others were downregulated (Additional file 8: Table S6). In LYSZa6 as compared to LYSZa5, 242 genes were differentially expressed, among which 114 were upregulated and others were downregulated (Additional file 9: Table S7). Heatmaps illustrating these differentially expressed genes (DEGs) display distinctive transcriptomic profiles between the progeny isolates and the ancestry isolates (Fig. 2A and B). We further clustered the isolates using Principal Coordinates Analysis (PCoA) based on Bray Curtis dissimilarity to check the differences between different groups. As seen from PCoA plots (Fig. 2C and D), LYSZa2 group and LYSZa5 group separated clearly from LYSZa3 group and LYSZa6 group respectively, indicating that the ancestry isolates and the progeny isolates possess distinct physiology. 74 common genes in total were found to be differentially regulated in both progeny isolates, LYSZa3 and LYSZa6 (Additional file 10: Table S8), indicating parallel changes evolved from same $P$. aeruginosa clone in different hosts during superinfection with SARS-CoV-2 virus.

We next performed Gene Ontology (GO) enrichment analysis using the DEGs to identify highly enriched functions in the progeny isolates (Fig. 3). 11 GO classes consisting of 7 biological processes, 3 molecular functions and 1 cellular component were enriched in LYSZa3 as compared to LYSZa2 (Fig. 3A). Whereas in LYSZa6 compared to LYSZa5, 13 GO classes consisting of 7 biological processes, 5 molecular functions and 
Table 1 Common genetic mutations identified between LYSZa3 and LYSZa6

\begin{tabular}{|c|c|c|c|c|c|c|c|c|c|}
\hline \multirow[b]{2}{*}{ Gene } & \multirow[b]{2}{*}{ Product } & \multicolumn{4}{|c|}{ LYSZa3 vs LYSZa2 } & \multicolumn{4}{|c|}{ LYSZa6 vs LYSZa5 } \\
\hline & & Type & $\begin{array}{l}\text { Amino acid } \\
\text { change }\end{array}$ & $\begin{array}{l}\text { Coding region } \\
\text { change in } \\
\text { longest } \\
\text { transcript }\end{array}$ & $\begin{array}{l}\text { Non- } \\
\text { synonymous }\end{array}$ & Type & $\begin{array}{l}\text { Amino acid } \\
\text { change }\end{array}$ & $\begin{array}{l}\text { Coding region } \\
\text { change in } \\
\text { longest } \\
\text { transcript }\end{array}$ & $\begin{array}{l}\text { Non- } \\
\text { synonymous }\end{array}$ \\
\hline PA0041 & $\begin{array}{l}\text { Hemagglu- } \\
\text { tinin }\end{array}$ & SNV & & $5803 C>T$ & No & SNV & & $5803 C>T$ & No \\
\hline \multirow[t]{8}{*}{ PA0259 } & \multirow{8}{*}{$\begin{array}{l}\text { Type } 6 \\
\text { lipase adap- } \\
\text { tor, Tla3 }\end{array}$} & SNV & & $333 A>G$ & No & SNV & & $333 A>G$ & No \\
\hline & & MNV & & 330_331delinsTT & No & MNV & & 330_331delinsTT & No \\
\hline & & SNV & & $327 \mathrm{~T}>\mathrm{G}$ & No & SNV & & $327 \mathrm{~T}>\mathrm{G}$ & No \\
\hline & & SNV & & $324 \mathrm{~T}>\mathrm{A}$ & No & SNV & & $324 \mathrm{~T}>\mathrm{A}$ & No \\
\hline & & MNV & Ser107Asn & 320_321delinsAT & Yes & MNV & Ser107Asn & 320_321delinsAT & Yes \\
\hline & & SNV & & $315 \mathrm{~T}>\mathrm{C}$ & No & SNV & & $315 \mathrm{~T}>\mathrm{C}$ & No \\
\hline & & SNV & Asp105Asn & $313 G>A$ & Yes & SNV & Asp105Asn & $313 G>A$ & Yes \\
\hline & & SNV & & $303 G>A$ & No & SNV & & $303 G>A$ & No \\
\hline \multirow[t]{18}{*}{ fiuA } & \multirow{18}{*}{$\begin{array}{l}\text { Ferrichrome } \\
\text { receptor } \\
\text { FiuA }\end{array}$} & SNV & & $1854 C>T$ & No & SNV & & $1854 C>T$ & No \\
\hline & & SNV & & $1842 G>C$ & No & SNV & & $1842 G>C$ & No \\
\hline & & SNV & & $1830 G>C$ & No & SNV & & $1830 G>C$ & No \\
\hline & & SNV & Ile608Met & $1824 C>G$ & Yes & SNV & Ile608Met & $1824 C>G$ & Yes \\
\hline & & SNV & Ile608Leu & $1822 \mathrm{~A}>\mathrm{C}$ & Yes & SNV & Ile608Leu & $1822 A>C$ & Yes \\
\hline & & SNV & & $1812 \mathrm{G}>\mathrm{A}$ & No & SNV & & $1812 \mathrm{G}>\mathrm{A}$ & No \\
\hline & & SNV & & $1800 G>C$ & No & SNV & & $1800 G>C$ & No \\
\hline & & SNV & & $1797 G>A$ & No & SNV & & $1797 G>A$ & No \\
\hline & & SNV & & $1794 G>C$ & No & SNV & & $1794 G>C$ & No \\
\hline & & SNV & & $1792 C>T$ & No & SNV & & $1792 C>T$ & No \\
\hline & & Deletion & Ser597fs & 1790delC & Yes & Deletion & Ser597fs & 1790delC & Yes \\
\hline & & SNV & Ser596Arg & $1788 \mathrm{~T}>\mathrm{A}$ & Yes & SNV & Ser596Arg & $1788 \mathrm{~T}>\mathrm{A}$ & Yes \\
\hline & & Insertion & Ser596fs & 1786dupA & Yes & Insertion & Ser596fs & 1786dupA & Yes \\
\hline & & SNV & Thr595Asn & $1784 C>A$ & Yes & SNV & Thr595Asn & $1784 C>A$ & Yes \\
\hline & & MNV & & $\begin{array}{l}\text { 1780_1781delin- } \\
\text { STC }\end{array}$ & No & MNV & & $\begin{array}{l}\text { 1780_1781delin- } \\
\text { STC }\end{array}$ & No \\
\hline & & Insertion & Asn593fs & 1778_1779insGG & Yes & Insertion & Asn593fs & 1778_1779insGG & Yes \\
\hline & & MNV & $\begin{array}{l}\text { Met592_- } \\
\text { Asn593de- } \\
\text { linslleTyr }\end{array}$ & $\begin{array}{l}\text { 1776_1777delin- } \\
\text { sAT }\end{array}$ & Yes & MNV & $\begin{array}{l}\text { Met592- } \\
\text { Asn593de- } \\
\text { linslleTyr }\end{array}$ & $\begin{array}{l}\text { 1776_1777delin- } \\
\text { sAT }\end{array}$ & Yes \\
\hline & & $\begin{array}{l}\text { Replace- } \\
\text { ment }\end{array}$ & Met592fs & 1773delinsCG & Yes & $\begin{array}{l}\text { Replace- } \\
\text { ment }\end{array}$ & Met592fs & 1773delinsCG & Yes \\
\hline \multirow[t]{4}{*}{ PA4514 } & \multirow{4}{*}{$\begin{array}{l}\text { Iron trans- } \\
\text { port outer } \\
\text { membrane } \\
\text { receptor }\end{array}$} & SNV & Thr193\|le & $578 C>T$ & Yes & SNV & Thr193\|le & $578 C>T$ & Yes \\
\hline & & $\begin{array}{l}\text { Replace- } \\
\text { ment }\end{array}$ & Asp190fs & 569_570delinsG & Yes & $\begin{array}{l}\text { Replace- } \\
\text { ment }\end{array}$ & Asp190fs & 569_570delinsG & Yes \\
\hline & & SNV & Gln189Leu & $566 \mathrm{~A}>\mathrm{T}$ & Yes & SNV & Gln189Leu & $566 \mathrm{~A}>\mathrm{T}$ & Yes \\
\hline & & Insertion & Gln189fs & 564dupG & Yes & Insertion & Gln189fs & 564dupG & Yes \\
\hline \multirow[t]{2}{*}{ PA5088 } & \multirow{2}{*}{$\begin{array}{l}\text { Type VI } \\
\text { secretion } \\
\text { lipase } \\
\text { immunity } \\
\text { protein, } \\
\text { Tli5b3 }\end{array}$} & SNV & Gly181Ser & $541 \mathrm{G}>\mathrm{A}$ & Yes & SNV & Gly181Ser & $541 G>A$ & Yes \\
\hline & & SNV & & $528 G>C$ & No & SNV & & $528 G>C$ & No \\
\hline
\end{tabular}

Full list of genetic mutations is included in Additional file 7: Table S5

1 cellular component were highly enriched (Fig. 3C). Among all of these enriched functions in both the progeny isolates, most significantly enriched biological processes are alginic acid biosynthetic process and protein secretion by the type VI secretion system (T6SS). All of 12 genes assigned to alginic acid biosynthetic 

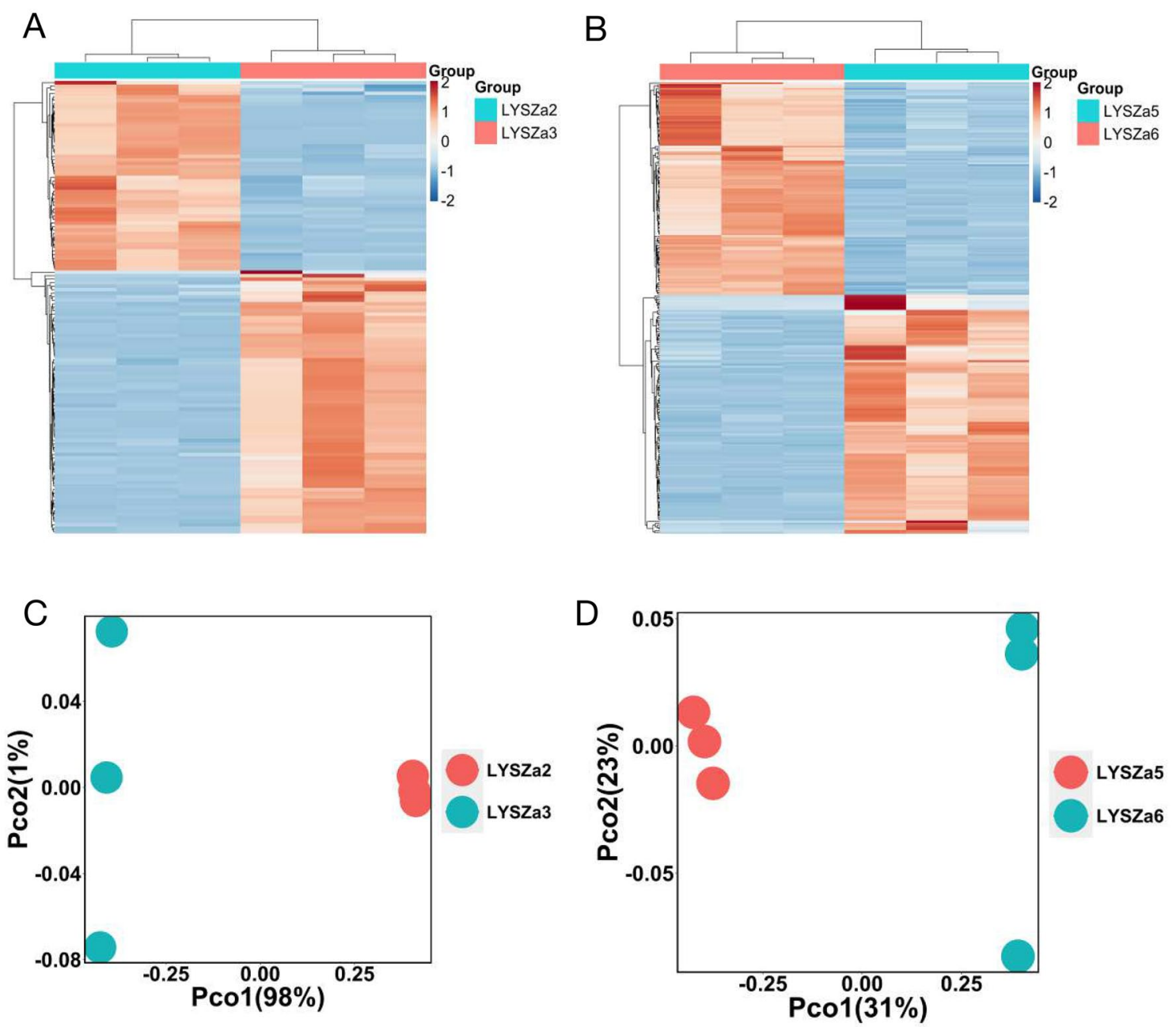

Fig. 2 Heatmaps and PCoA plots of DEGs between the ancestry isolates and the progeny isolates. A Heatmap of significant DEGs in LYSZa3 compared to LYSZa2; $\mathbf{B}$ heatmap of significant DEGs in LYSZa6 compared to LYSZa5; C PCoA plots of LYSZa3 group and LYSZa2 group based on DEGs using Bray Curtis dissimilarity; D PCoA plots of LYSZa6 group and LYSZa5 group based on DEGs using Bray Curtis dissimilarity

process $(\operatorname{alg} D, \operatorname{alg} X, \operatorname{alg} A, \operatorname{alg} E, \operatorname{alg} F, \operatorname{alg} L, \operatorname{alg} 44, \operatorname{alg}$, algK, alg8, algI, algG) were upregulated in LYSZa3 for 16.89 to 1091.52 folds (Table 2). The expression of same genes also increased significantly in LYSZa6 for 4.45 to 138.79 folds (Table 3). Eight genes assigned to protein secretion by T6SS, PA1657 (hsiB2), PA1658 (hsiC2), PA1659 (hsiF2), PA1660 (hsiG2), PA1661 (hsiH2), PA1662 ( $c l p V 2)$, PA1663 (sfa2), PA1666 (lip2), were all significantly downregulated in LYSZa3 for 4.46 to 6.47 folds (Table 2). In LYSZa6, beside the same eight genes mentioned above, seven other genes assigned to protein secretion by T6SS were also downregulated for 4.46 to 17.11 folds, including PA1656 (hsiA2), PA1665 (fha2), PA1667 (hsiJ2), PA1668 (dotU2), PA1669 (icmF2), stk1 and stp1 (Table 3). Besides these genes assigned to T6SS by GO enrichment analysis, the expression of several other genes involved in T6SS, $h c p B$, lip3 (PA2364), and dotU3 (PA2362), were also decreased in LYSZa3 (Table 2). While the expression of 15 other T6SS genes significantly decreased in LYSZa6, including clpV1, PA3904 (PAAR4), PA2702 (tse2), PA2774 (tse4), PA2775 (tsi4), vgrG1, PA0093 (tse6), PA3905 (tecT), PA0082 (tssA1), PA2703 (tsi2), PA0094 (eagT6), PA3484 (tse3), PA5266 ( $v g r G 6), h c p A$ and $h c p B$ (Table 3). P. aeruginosa carries three sets of T6SS systems, HSI-I, HSI-II and HSI-III respectively, to regulate its virulence to bacterial neighbors and host cells. T6SS is a phage-like structure injecting exotoxins to neighboring cells and consists of several key structural components including sheath and baseplate components encoded by tss/hsi genes, spike proteins encoded by $\operatorname{vgr} G$ genes, syringe proteins encoded by 

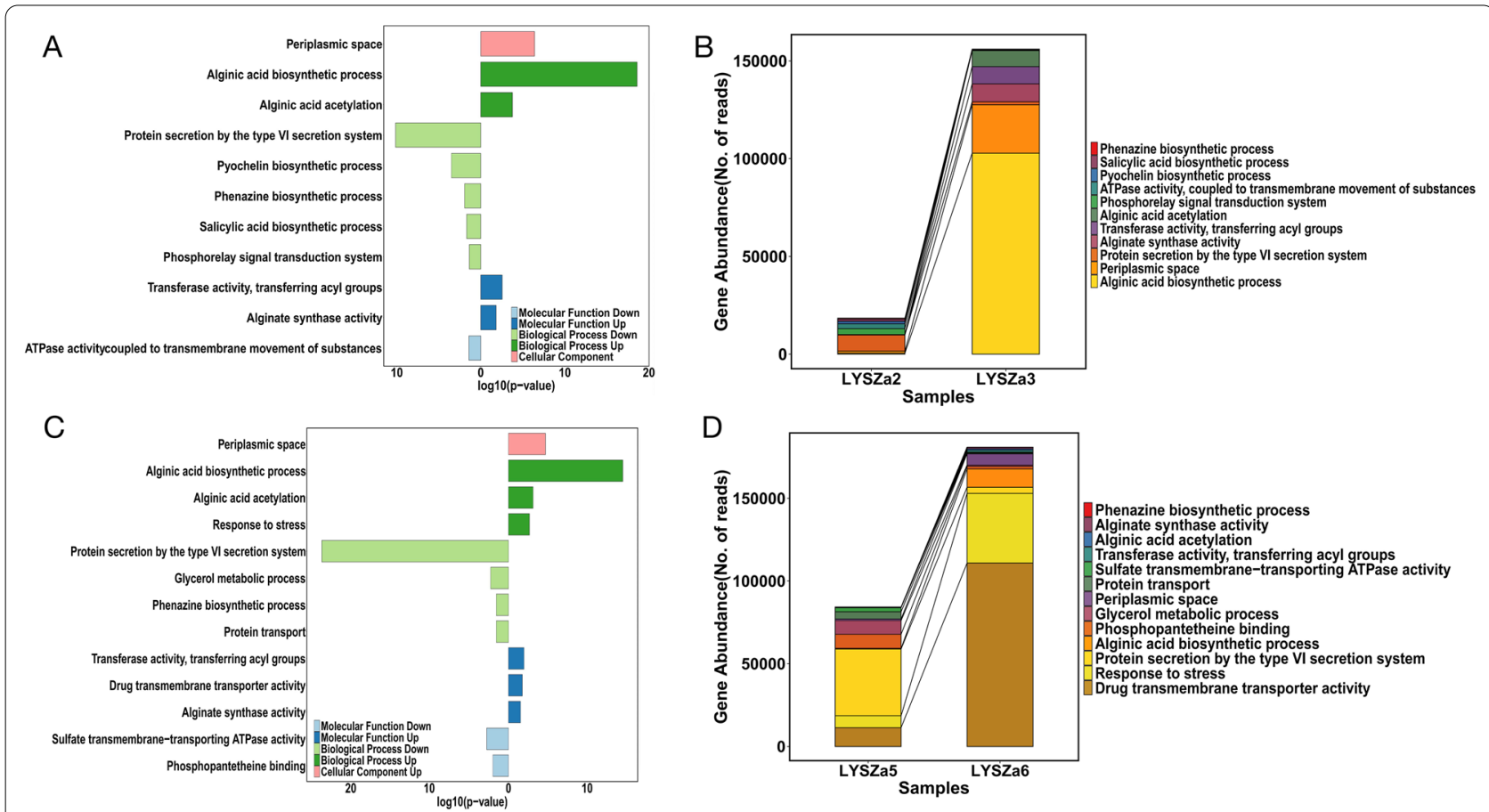

Fig. 3 GO enrichment analysis and variation in gene abundance in each GO. A GO enriched in LYSZa3 compared to LYSZa2, bars towards right-hand side showing genes assigned to the functions were upregulated, bars towards left-hand side showing genes assigned to the functions were downregulated, up- and down-regulation are indicated in the legend as well, $p$-value $<0.05$; $\mathbf{B}$ Variation in gene abundance involved in the enriched GO functions listed in A in LYSZa2 and LYSZa3; C GO enriched in LYSZa6 compared to LYSZa5, bars towards right-hand side showing genes assigned to the functions were upregulated, bars towards left-hand side showing genes assigned to the functions were downregulated, up- and down-regulation are indicated in the legend as well, $p$-value $<0.05$; $\mathbf{D}$ Variation in gene abundance involved in the enriched GO functions listed in $C$ in LYSZa5 and LYSZa6

$h c p$ genes, ATPases encoded by $c l p V$ genes and etc. As illustrated in Fig. 4, the downregulated genes in both progeny isolates, especially those of LYSZa6, are mostly involved in HSI-II gene cluster with several others scattered on another two gene clusters. The higher number of DEGs observed in LYSZa6 as compared to LYSZa3 is likely due to the longer evolving time of LYSZa6 within the host. The changes in the gene abundance assigned to different GO functions are illustrated in Fig. 3B and D. There was a dramatic increase in the abundance of genes involved in alginic acid biosynthetic process and significant decrease in the abundance of genes involved in protein secretion by T6SS in the progeny isolates. Such observation conveyed excessive biosynthesis of alginate and suppression of T6SS in the progeny isolates during colonization. Besides T6SS, T3SS is another important determinant for $P$. aeruginosa virulence to infect host cells. The results of RNA-Seq indicated that most of the T3SS genes expressed at low levels in all four isolates without significant differences between the progeny isolates and the ancestor isolates (data not shown). To confirm such observation, we selected few genes encoding for different structural components of T6SS ( $v g r G 1$ for needle tip, $h c p A$ for secreted protein, hsiC2 for sheath component and clpV2 for ATPase) and T3SS (popD for translocation apparatus, $p s c F$ for needle filament and $p s c N$ for ATPase complex) for RTPCR examination. The results of RT-PCR tests (Additional file 2: Fig. S2) showed that the expression level of T6SS genes indeed decreased in the progeny isolates while the expression of T3SS genes remained low and insignificantly differentiated between the progeny and the ancestor. Though the decrease in expression of $p s c F$ gene in LYSZa6 needed to be further studied, such results suggested that T3SS plays minimal role in regulating the virulence of the isolates towards host cells.

In accordance with the gene expression analysis, the progeny isolates produce excessive alginate and exhibit more mucoid phenotype after in vitro cultivation on agar plates (Fig. 5A). As alginate plays an important role in biofilm architecture and development [23], we thus quantified biofilm formation of the isolates in vitro by crystal violet staining method. As seen from Fig. 5B, aligned with gene expression data, an increase in the 
Table 2 Selected DEGs in LYSZa3 compared to LYSZa2 related to alginate biosynthesis and type VI secretion system

\begin{tabular}{|c|c|c|c|c|c|c|}
\hline Gene & Product name & Base mean & Fold change & Adj.p-value & LYSZa2 Mean & LYSZa3 Mean \\
\hline $\operatorname{alg} D$ & GDP-mannose 6-dehydrogenase AlgD & $27,877.94$ & 1091.52 & $0.00 \mathrm{E}+00$ & 61.33 & $50,065.00$ \\
\hline $\operatorname{alg} x$ & Alginate biosynthesis protein AlgX & 1967.25 & 256.42 & $2.31 \mathrm{E}-236$ & 18.33 & 3478.67 \\
\hline $\operatorname{alg} A$ & $\begin{array}{l}\text { Phosphomannose isomerase/guanosine 5'-diphospho-D- } \\
\text { mannose pyrophosphorylase }\end{array}$ & 5853.40 & 238.02 & $0.00 \mathrm{E}+00$ & 59.00 & $10,382.00$ \\
\hline algE & Alginate production outer membrane protein AlgE precursor & 2204.36 & 221.80 & $3.29 \mathrm{E}-252$ & 24.00 & 3889.33 \\
\hline algF & Alginate o-acetyltransferase AlgF & 1648.75 & 211.59 & $3.00 E-242$ & 18.67 & 2924.00 \\
\hline $\operatorname{algl}$ & Poly(beta-D-mannuronate) lyase precursor AlgL & 2134.58 & 182.28 & $4.64 \mathrm{E}-245$ & 28.00 & 3766.33 \\
\hline $\operatorname{alg} 44$ & Alginate biosynthesis protein Alg44 & 1855.41 & 162.63 & $1.64 \mathrm{E}-285$ & 27.33 & 3260.33 \\
\hline alg & Alginate o-acetyltransferase AlgJ & 1033.36 & 154.76 & $2.12 \mathrm{E}-191$ & 16.00 & 1783.33 \\
\hline $\operatorname{algk}$ & alginate biosynthetic protein AlgK precursor & 1171.78 & 145.20 & $2.18 \mathrm{E}-233$ & 19.33 & 2043.00 \\
\hline alg8 & Alginate biosynthesis protein Alg8 & 2758.04 & 133.33 & $0.00 \mathrm{E}+00$ & 49.67 & 4939.33 \\
\hline algl & Alginate o-acetyltransferase Algl & 1457.21 & 59.29 & $1.83 \mathrm{E}-259$ & 58.33 & 2501.67 \\
\hline $\operatorname{alg} G$ & Alginate-c5-mannuronan-epimerase AlgG & 1682.20 & 16.89 & $1.73 E-125$ & 226.00 & 2793.33 \\
\hline$h с p B$ & Secreted protein Hcp & 107.95 & -4.46 & $1.17 \mathrm{E}-20$ & 215.67 & 36.00 \\
\hline PA1663 & $\mathrm{Sfa} 2$ & 305.43 & -4.46 & $2.74 \mathrm{E}-47$ & 603.33 & 100.00 \\
\hline PA1666 & Lip2 & 159.33 & -4.50 & 2.97E-24 & 319.00 & 52.67 \\
\hline PA2362 & DotU3 & 22.49 & -4.54 & 4.95E-06 & 44.00 & 7.00 \\
\hline PA1660 & HsiG2 & 346.81 & -4.63 & $1.56 \mathrm{E}-59$ & 686.67 & 110.33 \\
\hline PA1662 & clpV2 & 1014.01 & -4.63 & $3.41 \mathrm{E}-44$ & 2020.67 & 318.00 \\
\hline PA1661 & $\mathrm{HsiH} 2$ & 246.25 & -4.65 & $1.19 E-39$ & 488.33 & 77.67 \\
\hline PA1659 & HsiF2 & 163.52 & -5.59 & $1.38 \mathrm{E}-34$ & 334.00 & 43.00 \\
\hline PA1657 & HsiB2 & 842.21 & -6.21 & $6.69 \mathrm{E}-109$ & 1743.67 & 204.33 \\
\hline PA1658 & $\mathrm{HsiC2}$ & 1839.66 & -6.47 & $4.92 \mathrm{E}-141$ & 3824.67 & 437.00 \\
\hline PA2364 & Lip3 & 32.22 & -9.20 & $6.01 E-13$ & 70.33 & 5.67 \\
\hline
\end{tabular}

Filtering criteria are fold change $\geqq 4$, adjusted $p$-value $<0.05$ and base mean $\geqq 20$. Full list of DEGs is included in Additional file 8: Table S6

biofilm formation was observed from LYSZa3 and LYSZa6 compared to LYSZa2 and LYSZa5 respectively.

\section{Suppression of T6SS of $P$. aeruginosa during adaptation in COVID-19 patients}

T6SS is used as a weapon by $P$. aeruginosa to compete with neighboring bacteria cells and to combat with the host cells in order to gain surviving advantage in the polymicrobial environment in the respiratory system. Suppression in T6SS would reduce its advantage to outcompete other bacteria and attenuate its virulence to escape from host immune clearance. We thus performed in vitro bacterial competition test and ex vivo cytotoxicity assay of the isolates to confirm the changes in the anti-prokaryotic and anti-eukaryotic capability between the ancestry isolates and the progeny isolates.

We firstly examined the capability of the $P$. aeruginosa isolates in killing the model pray organism E. coli through bacterial competition assay. The $P$. aeruginosa isolates were mixed and cultured with E. coli/placZ at 1:1 ratio respectively. The mixtures were diluted to $10^{-3}$ while triplicates of each dilution were then spotted and cultured on plates containing $\mathrm{X}$-gal to test the killing efficiency
(Fig. 6A and B). E. coli/pLacZ strain was able to digest $\mathrm{X}$-gal and appeared as blue colonies on plates. Survival of $E$. coli was determined both qualitatively by visualizing the intensity of blue pigment of the colonies and quantitatively by counting the blue $E$. coli $\mathrm{CFU}$ left. As observed from Fig. 6A and $\mathrm{B}$, almost no trace of blue pigment could be seen from the mixture of E. coli and the ancestry $P$. aeruginosa isolates, LYSZa2 and LYSZa5. More intensive blue colonies were observed from the mixed cultures of E. coli and the progeny isolates, LYSZa3 and LYSZa6, indicating disadvantage of the progeny isolates to outcompete $E$. coli compared to their respective ancestry isolates. Same trend was also observed from quantitative tests illustrated in Fig. $6 \mathrm{C}$ where higher numbers of $E$. coli CFU were left after coculturing with LYSZa3 and LYSZa6 comparing with those culturing with LYSZa2 and LYSZa5 respectively. Such results indicated a decrease in the bactericidal activity of the progeny isolates during bacterial competition consequently due to the inhibition of T6SS.

We then performed ex vivo macrophage killing assay to assess the $P$. aeruginosa cytotoxicity against the eukaryotic cells. Cultures of the P. aeruginosa isolates 
Table 3 Selected DEGs in LYSZa6 compared to LYSZa5 related to alginate biosynthesis and type VI secretion system

\begin{tabular}{|c|c|c|c|c|c|c|}
\hline Gene & Product name & Base mean & Fold change & $\begin{array}{l}\text { Adj } \\
\text { p-value }\end{array}$ & LYSZa2 Mean & LYSZa3 Mean \\
\hline $\operatorname{alg} D$ & GDP-mannose 6-dehydrogenase AlgD & 2956.90 & 138.79 & $2.47 \mathrm{E}-170$ & 39.33 & 6375.00 \\
\hline $\operatorname{alg} x$ & Alginate biosynthesis protein AlgX & 271.80 & 45.97 & $1.05 \mathrm{E}-75$ & 10.67 & 585.67 \\
\hline algL & Poly(beta-D-mannuronate) lyase precursor AlgL & 283.61 & 44.85 & $1.98 \mathrm{E}-74$ & 11.33 & 609.00 \\
\hline alg & Alginate o-acetyltransferase Alg」 & 118.03 & 32.97 & 3.93E-33 & 6.33 & 248.67 \\
\hline $\operatorname{alg} E$ & Alginate production outer membrane protein AlgE precursor & 254.48 & 25.19 & $1.11 \mathrm{E}-61$ & 18.33 & 535.33 \\
\hline $\operatorname{alg} A$ & $\begin{array}{l}\text { Phosphomannose isomerase/guanosine 5'-diphospho- } \\
\text { D-mannose pyrophosphorylase }\end{array}$ & 481.88 & 21.52 & $9.22 \mathrm{E}-64$ & 39.00 & 1000.67 \\
\hline algF & Alginate o-acetyltransferase AlgF & 152.00 & 18.68 & $7.50 \mathrm{E}-42$ & 14.33 & 314.67 \\
\hline alg 8 & Alginate biosynthesis protein Alg8 & 292.51 & 14.91 & 1.09E-59 & 34.67 & 599.33 \\
\hline $\operatorname{alg} 44$ & Alginate biosynthesis protein Alg44 & 195.56 & 14.43 & $3.38 \mathrm{E}-61$ & 23.67 & 403.00 \\
\hline $\operatorname{algk}$ & Alginate biosynthetic protein AlgK precursor & 135.65 & 10.35 & $6.37 \mathrm{E}-39$ & 21.67 & 272.67 \\
\hline algl & Alginate o-acetyltransferase Algl & 218.14 & 8.40 & $7.47 \mathrm{E}-47$ & 43.00 & 427.33 \\
\hline $\operatorname{alg} G$ & Alginate-c5-mannuronan-epimerase AlgG & 356.62 & 4.45 & 4.15E-53 & 121.33 & 644.33 \\
\hline clpV1 & ClpV1 & 5315.67 & -4.08 & $7.48 \mathrm{E}-33$ & 7854.33 & 2309.33 \\
\hline PA3904 & PAAR4 & 1457.06 & -4.17 & $3.79 \mathrm{E}-33$ & 2178.00 & 631.67 \\
\hline PA2702 & Tse2 & 280.90 & -4.35 & $2.50 \mathrm{E}-29$ & 419.33 & 117.33 \\
\hline PA2774 & Tse4 & 296.61 & -4.37 & $4.85 \mathrm{E}-26$ & 461.67 & 122.33 \\
\hline PA2775 & Tsi4 & 133.81 & -4.71 & 7.24E-19 & 212.67 & 52.33 \\
\hline $\operatorname{vgrG1}$ & VgrG1 & 2637.35 & -4.74 & $1.56 \mathrm{E}-50$ & 3969.33 & 1016.67 \\
\hline PA0093 & Tse6 & 947.79 & -4.91 & $3.88 \mathrm{E}--46$ & 1469.00 & 360.00 \\
\hline PA3905 & Type VI effector chaperone for Tox-Rease, TecT & 1073.56 & -4.95 & $1.10 \mathrm{E}-53$ & 1671.00 & 403.00 \\
\hline PA0082 & TsSA1 & 1449.46 & -5.32 & $1.76 \mathrm{E}-55$ & 2265.00 & 515.00 \\
\hline PA2703 & Tsi2 & 98.26 & -5.56 & $5.99 \mathrm{E}-24$ & 156.67 & 33.00 \\
\hline PA0094 & EagT6 & 251.06 & -5.79 & 1.43E-38 & 401.67 & 82.00 \\
\hline PA3484 & Tse3 & 544.56 & -5.86 & $1.50 \mathrm{E}-54$ & 869.33 & 174.33 \\
\hline stk1 & Stk1 & 263.85 & -5.90 & $1.38 \mathrm{E}-56$ & 423.00 & 84.67 \\
\hline PA5266 & VgrG6 & 396.48 & -6.81 & $6.50 \mathrm{E}-47$ & 654.33 & 113.33 \\
\hline PA1667 & HsiJ2 & 678.54 & -6.99 & $2.41 \mathrm{E}-100$ & 1119.67 & 188.67 \\
\hline PA1656 & $\mathrm{HsiA2}$ & 3257.03 & -7.01 & $4.84 \mathrm{E}-80$ & 5349.67 & 911.33 \\
\hline PA1662 & clpV2 & 2538.08 & -8.98 & $3.88 \mathrm{E}-108$ & 4332.33 & 564.33 \\
\hline PA1661 & $\mathrm{HsiH} 2$ & 693.51 & -9.91 & $1.55 \mathrm{E}-110$ & 1186.67 & 142.00 \\
\hline PA1666 & Lip2 & 340.97 & -10.48 & $2.48 \mathrm{E}-72$ & 589.00 & 65.67 \\
\hline stp 1 & Stp1 & 327.95 & -10.61 & $1.67 \mathrm{E}-70$ & 562.67 & 62.67 \\
\hline$h с p A$ & Secreted protein Hcp & 72.34 & -10.75 & $7.25 \mathrm{E}-26$ & 125.33 & 13.67 \\
\hline PA1665 & Fha2 & 840.73 & -10.96 & $1.12 \mathrm{E}-108$ & 1456.67 & 155.33 \\
\hline PA1660 & HsiG2 & 907.03 & -11.05 & $1.78 \mathrm{E}-125$ & 1551.67 & 167.33 \\
\hline PA1669 & IcmF2 & 2052.74 & -11.08 & $6.20 \mathrm{E}-107$ & 3547.33 & 375.67 \\
\hline$h с p B$ & Secreted protein $\mathrm{Hcp}$ & 204.27 & -11.30 & $8.81 E-47$ & 358.00 & 36.67 \\
\hline PA1668 & DotU2 & 487.58 & -11.30 & $1.39 \mathrm{E}-105$ & 836.33 & 88.00 \\
\hline PA1663 & $\mathrm{Sfa} 2$ & 883.17 & -12.92 & 4.47E-74 & 1556.67 & 139.33 \\
\hline PA3334 & Acp3 & 236.04 & -13.79 & $1.82 \mathrm{E}-73$ & 415.67 & 35.33 \\
\hline PA1657 & HsiB2 & 2539.11 & -14.39 & $1.32 \mathrm{E}-112$ & 4445.00 & 366.00 \\
\hline PA1659 & HsiF2 & 480.65 & -17.06 & $4.25 \mathrm{E}-89$ & 843.33 & 59.00 \\
\hline PA1658 & $\mathrm{HsiC2}$ & 5746.91 & -17.11 & $4.82 \mathrm{E}-66$ & $10,179.00$ & 695.67 \\
\hline
\end{tabular}


HSI-I

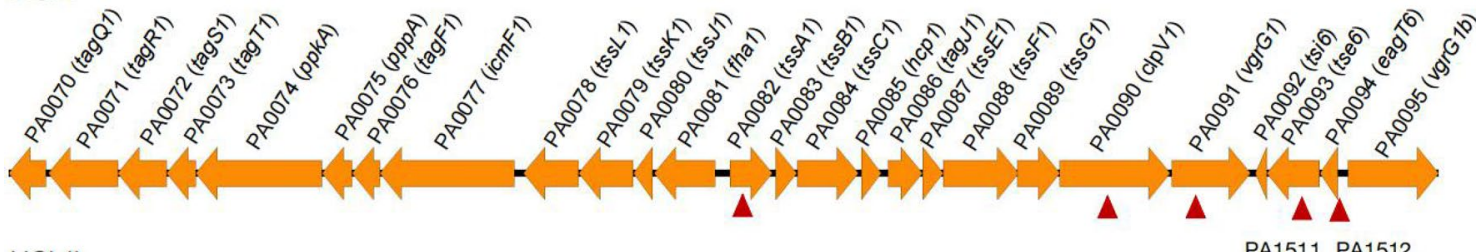

HSI-II

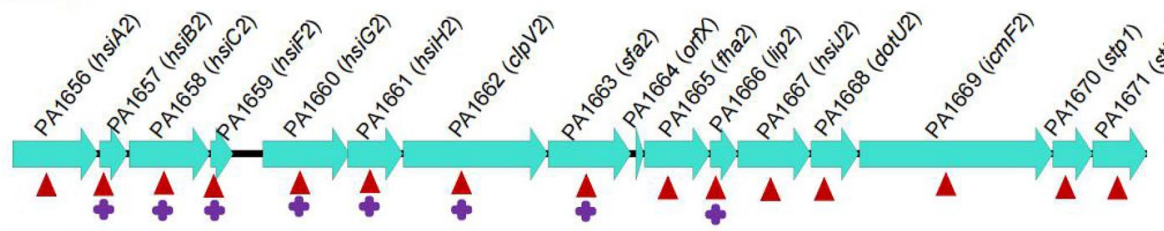

PA1511 PA1512

(vgrG2a) (hcpA)

HSI-III

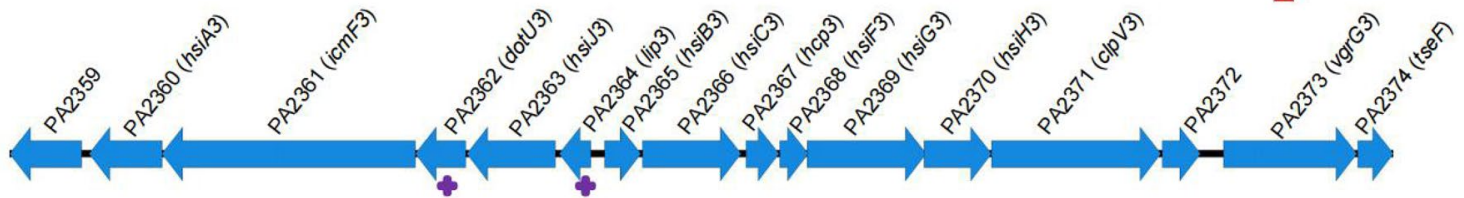

Fig. 4 Schematic figure of HSI-I, HSI-II and HSI-III gene clusters. Genes downregulated in LYSZa3 are denoted by purple crosses; genes downregulated in LYSZa6 are denoted by red triangles. Gene name was obtained from Pseudomonas Genome Database [49]

A
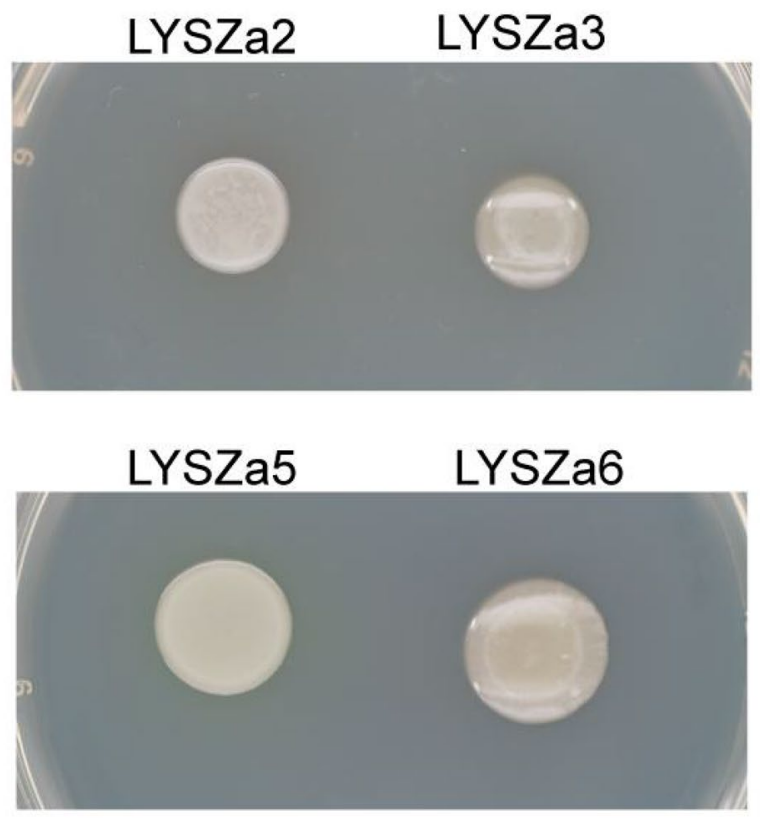

B
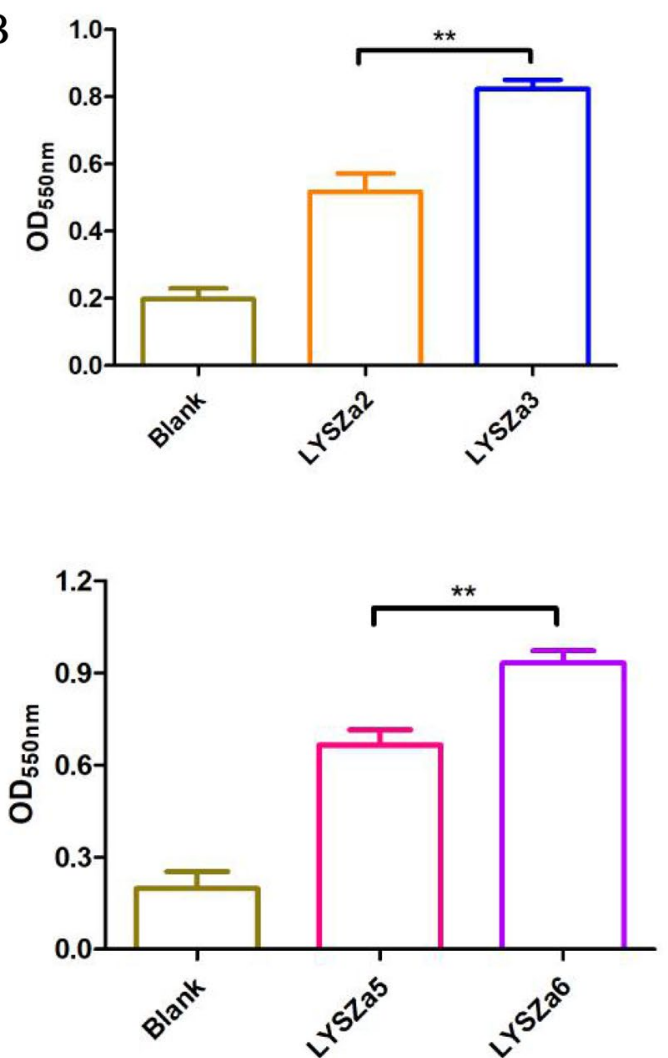

Fig. 5 Phenotypic analysis. A Colony morphologies of the isolates; B Biofilm quantification tests by crystal violet staining, ${ }^{* *} p-$ value $<0.05$; Upper panel: biofilm quantification test of LYSZa2 and LYSZa3, lower panel: biofilm quantification test of LYSZa5 and LYSZa6 

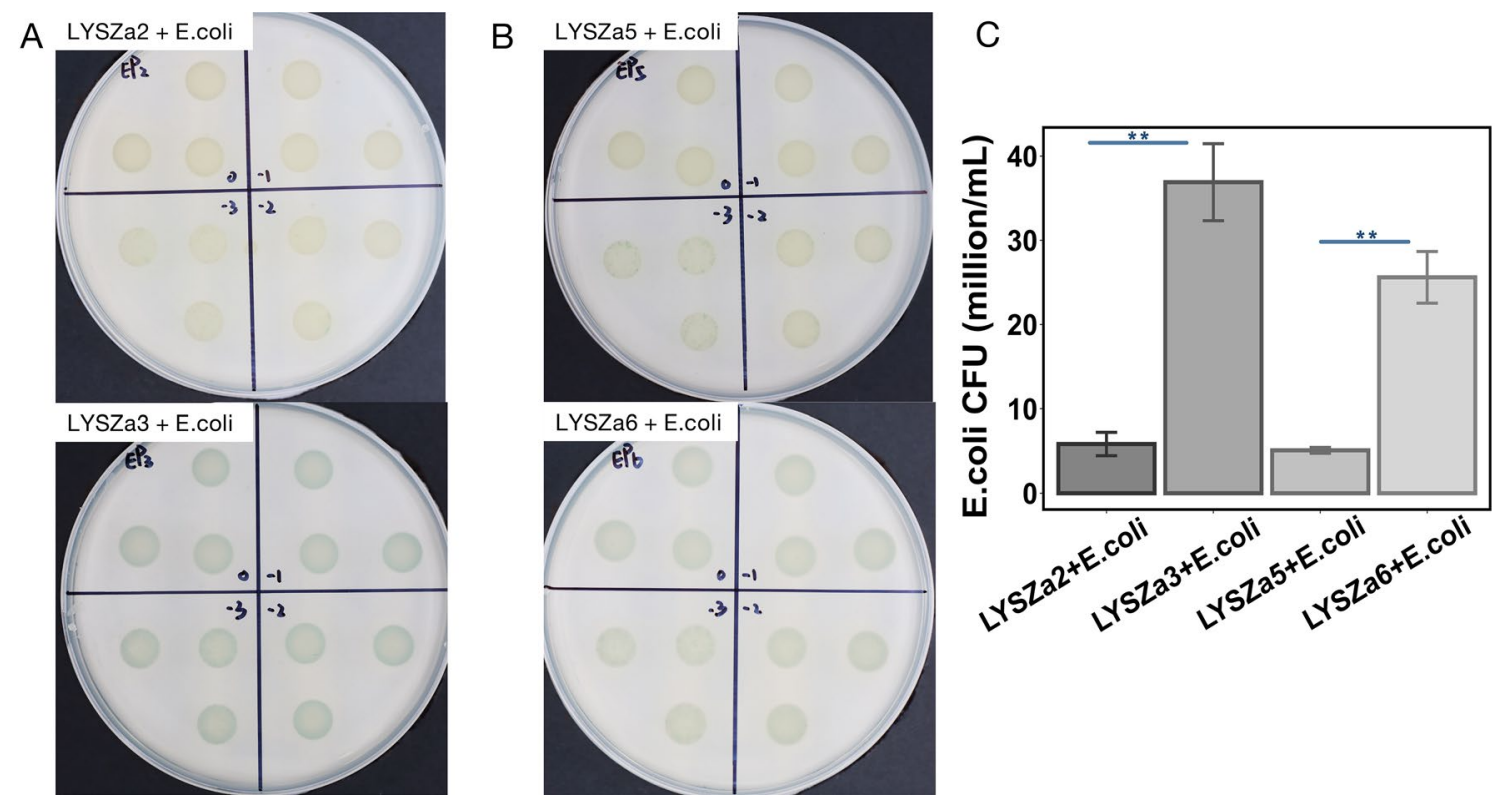

Fig. 6 Bacterial competition assay between the isolates and E. coli. Blue pigment intensity indicates the survival of E. coli. A Upper panel: competition between LYSZa2 and E. coli; lower panel: competition between LYSZa3 and E. coli, number in each section denotes the dilution factor, ranged from $10^{0}$ to $10^{-3}$; B Upper panel: competition between LYSZa5 and E. coli; lower panel: competition between LYSZa6 and E. coli, number in each section denotes the dilution factor, ranged from $10^{0}$ to $10^{-3}$; C Counts of E. coli CFU left in each competition, ${ }^{* *} p$-value $<0.05$

were added to infect RWA264.7 macrophages individually. Relative lactate dehydrogenase $(\mathrm{LDH})$ release was measured to determine the death of macrophages. As illustrated in Fig. 7A, LDH released by macrophages infected by LYSZa3 was much lower than that of LYSZa2. Similar results were observed from LYSZa5 and LYSZa6 where macrophages infected by LYSZa5 released a significant higher level of LDH comparing to LYSZa6. Thus, both LYSZa3 and LYSZa6 strains possess weaker cytotoxicity comparing to their ancestral isolates. Macrophages play an important role in the defense against the invading pathogens in the host, the reactive oxygen species (ROS) produced by macrophages within phagolysosome contributes to the destruction and clearance of pathogens [24]. Next, we have measured the intracellular ROS production in macrophages after $3 \mathrm{~h}$ infection of LYSZa2, LYSZa3, LYSZa5 and LYSZa6. We found that the ROS level within the macrophages which infected with the progeny strains were lower than the ancestor strains (Fig. 7B and C). Such results suggested that these progeny isolates possess attenuated virulence towards their
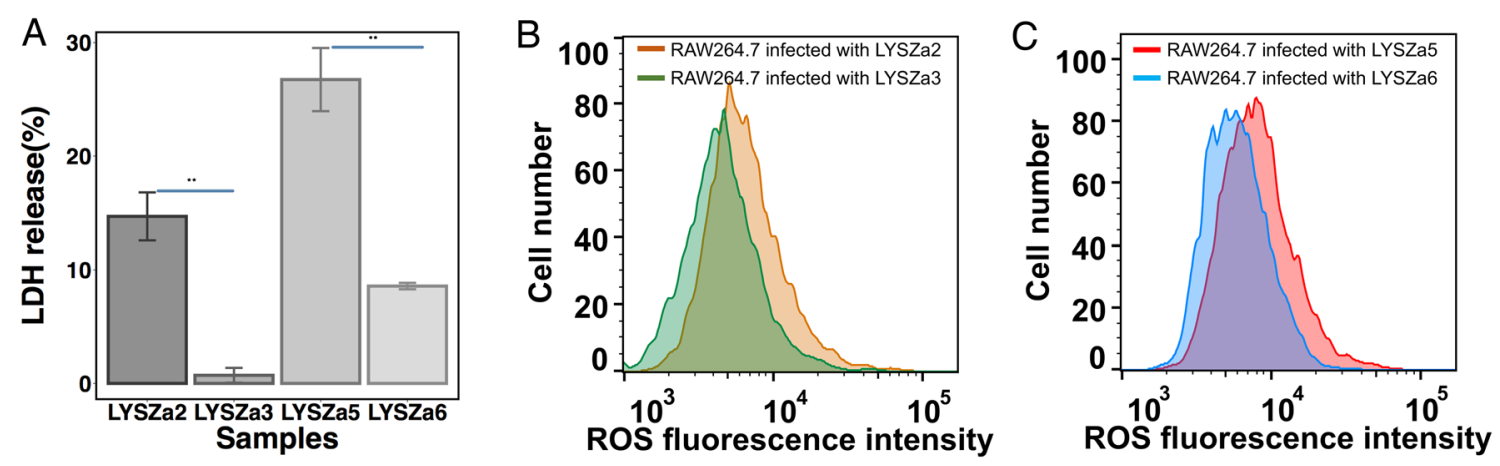

Fig. 7 Macrophage cytotoxicity and ROS production of the ancestry isolates and the progeny isolates. A The cytotoxicity effect against macrophage cells of LYSZa2, LYSZa3, LYSZa5 and LYSZa6 is illustrated by the percent of LDH released, ${ }^{* *} p$-value $<0.05$. B The intracellular ROS levels in RAW264.7 cells at $3 \mathrm{~h}$ after phagocytosis of LYSZa2, LYSZa3 and LYSZa5, LYSZa6 (C) 
eukaryotic hosts after short period of adaptation in the COVID-19 patients.

\section{Discussion}

It is well known that secondary bacterial infection after virus (e.g. influenza) infection will further increase morbidity and mortality due to virus infection. Even though a lot of knowledge regarding the lung immunology and SARS-CoV-2 virus pathogenesis has been provided recently, the increased occurrence of illness and death due to bacterial superinfection and its complications indicated that there is a much underestimated and neglected influence of bacterial superinfection on the disease progression in COVID-19 patients. In this study, we isolated the ancestor and the progeny isolate pairs of hospital-acquired $P$. aeruginosa strains from two critically ill COVID-19 patients to investigate its adaptive evolution during superinfection with SARS-CoV-2 virus. Our results suggested that $P$. aeruginosa upregulates its alginate biosynthesis and downregulates its T6SS to persist in the respiratory system and reduce its virulence to escape from host clearance for longer colonization. To the best of our knowledge, this is the first study describing the adaptive evolution of $P$. aeruginosa superinfection within the COVID-19 patients, which suggests that SARS-CoV-2 virus infection could provide a lung environment that allows rapid change of bacterial pathogens with genetic traits suitable for chronic infections.

The $P$. aeruginosa isolates collected from the two patients belongs to the same MLST type. In addition, the two patients lived in the same ward during hospitalization, they are therefore hospital-acquired $P$. aeruginosa strain. Genomic sequencing analysis revealed that the genomes of the isolates carry specific GIs for DNA repair and protein secretion systems, and ARGs for multiple drug classes. Common genomic modification events identified between the progeny isolates and the ancestral isolates indicated the genomic changes in genes related to T6SS, tla3 and tli5b3. Through differential gene expression analysis, we found that genes involved in alginate biosynthesis were upregulated greatly while genes involved in T6SS protein secretion system were significantly downregulated. Alginate is an essential EPS component converting non-mucoid $P$. aeruginosa to mucoid phenotype, and is important for biofilm structure and antimicrobial resistance [23]. Clinical $P$. aeruginosa strains overproducing alginate isolated from CF patients enhanced biofilm formation leading to the aggravation of disease conditions and poor prognosis [25, 26]. Moreover, excessive biosynthesis of alginate in $P$. aeruginosa promotes its superinfection with other pathogens such as $S$. aureus and B. cenocepacia and increases their persistence in CF infections $[27,28]$. Overproduction of alginate and increased biofilm formation were observed in the progeny strains in this study, inferring the potential mechanism adopted by P. aeruginosa in COVID-19 patients to enhance colonization and promote superinfection. As mucoid P. aeruginosa upon overexpression of alginate recalcitrating against antibiotics is well described $[23,29,30]$, thick alginate layer is also probably a key contributing factor to the increase in the antimicrobial resistance of the progeny isolate observed. Excessive alginate may also facilitate coexistence of $P$. aeruginosa and other superinfecting pathogens for secondary bacterial infection, and alter the microecological environment in the respiratory system of COVID-19 patients. Further analysis is needed for deeper investigation on the role of alginate in the microbial community in COVID-19 patients.

A more notable observation is the attenuation of T6SS in the $P$. aeruginosa isolates during short term evolution in COVID-19 patients. The expression of genes involved in T6SS were significantly downregulated in both the progeny isolates. $P$. aeruginosa T6SS functions as a phage-like toxin delivery apparatus to transport toxic effectors into surrounding competing bacterial cells and host cells to gain survival advantages and infect host cells [31]. P. aeruginosa carries three types of T6SS, being HSII, HSI-II, and HSI-III respectively. HSI-I is dedicated to inter-bacterial competition while HSI-II and HSI-III associate with both anti-prokaryotic and anti-eukaryotic functions [32, 33]. All of these three gene clusters contain $h c p, c l p V$, vgrG, tss/hsi genes and other genes including $p p k A, p p p A, t a g, s f a, f h a, l i p, \operatorname{dot} U, i c m F, s t p$ and $s t k$ genes for the full function of T6SS apparatus (Fig. 4) [31, 34]. In both of the $P$. aeruginosa progeny isolates, especially in LYSZa6, the expression of most of HSI-II genes and associated genes were downregulated significantly (Fig. 4). Such results indicated a general inhibition of HSI-II T6SS in the P. aeruginosa isolates during adaptation and evolution in the patients to adapt to COVID-19 environment. As HSI-II T6SS associates with anti-bacterial and anti-host functions, we tested both the bacterial killing capacity and macrophage cytotoxicity of the isolates. In addition to T6SS, T3SS is another key virulence regulating factor in $P$. aeruginosa, translocating exotoxins to infect host cells. Our RNA-Seq and RT-PCR results suggested a low and unchanged T3SS activity in all the isolates, indicating minimal contribution from T3SS to the attenuation of virulence of the progeny isolates to host cells. Moreover, previous study has indicated that P. aeruginosa T3SS associates with its planktonic phenotype and acute infection while T6SS is associated with biofilm phenotype and chronic infection [35]. Our results showed $P$. aeruginosa forms more biofilm through upregulation of alginate biosynthesis and reduced virulence 
through attenuation of T6SS. These results confirmed the decrease in the virulence to both neighboring bacteria and host cells of the progeny isolates due to T6SS attenuation. Previous studies had demonstrated that $P$. aeruginos a abrogated its T6SS to adapt to the host environment by genetic modification on genes such as $v g r G$ and $v g r G 4$ during chronic infection of cystic fibrosis [17, 36]. Here we showed that $P$. aeruginosa attenuated its T6SS at transcriptional level to gain fitness and survival advantages during acute infection of COVID-19 pneumonia.

The attenuation of T6SS in $P$. aeruginosa may give opportunities to other bacterial species to thrive in the host lung. A recent research showed that $P$. aeruginosa abrogating its T6SS could be outcompeted by coinfecting B. cenocepaica in CF patients in an age- and T6SS-dependent manner and predisposing host to superinfection of $B$. cenocepacia [37]. Thus, $P$. aeruginosa attenuating its T6SS in COVID-19 patients could probably play a pivotal role in coexisting with other pathogenic strains in the polymicrobial environment and promoting subsequent infection in COVID-19 patients. However, metagenomics analysis of the sputum of the two patients in which the $P$. aeruginosa isolate LYSZa2, LYSZa3, LYSZa5, LYSZa6 were sampled indicated that there is no change in the microbial diversity during the short colonization period of $P$. aeruginosa (Additional file 1: Fig. S1). Moreover, $P$. aeruginosa adaptation described in this study is rather disease-specific since it adapts to the lung environments differently in other disease such as cystic fibrosis and ventilated associated pneumonia. Previous studies have shown that $P$. aeruginosa altered its quorum sensing systems and cell wall component (lasR, $m p l)$ to reduce its virulence during adaptation in the airway of VAP patients[16]; while this bacterium slowly adapts to the airway of CF patients by genetic mutations in its $m u c A, \operatorname{alg} U, v g r G$ and etc. genes for chronic infection $[17,38]$. To the best of our knowledge, the attenuation of P. aeruginosa HSI-II T6SS gene expression in COVID-19 patients has not been reported in other studies. Moreover, isolates collected from different patients exhibited similar gene expression pattern. Thus, $P$. aeruginosa adaptation described in this study is rather disease-specific. Our results suggest that the attenuation of T6SS in $P$. aeruginosa in COVID-19 patients might gain some advantage in escaping from the host immune clearance, which needs further exploration.

In this study, we have also examined the microbiome in the lungs of the two COVID-19 patients enrolled in this study. Result of metagenomics analysis indicated that $P$. aeruginosa is the most abundant species in the lungs, followed by Ralstonia pickettii, Cellvibrio japonicus, Botrytis cinerea and $S$. aureus. The difference in the respiratory microbial composition between COVID-19 patients with and without VAP is insignificant while the dominating species are Klebsiella spp., P. aeruginosa, S. maltophilia, S. aureus etc. [39]. A study conducted on the core microbiome in the airways of patients with different pulmonary diseases including chronic obstructive pulmonary disease(COPD), exacerbated chronic obstructive pulmonary disease (ECOPD), sarcoidosis and interstitial lung diseases(ILD) showed that the most abundant taxa in each disease is Reyranella, Serratia, Serratia and Haemophilus respectively. Other dominating taxa include Prevotella, Escherichia_Shigella, Streptococcus, Pseudomonas and etc. with varied abundance in different diseases [40]. Another recent study analyzing the core microbiome in the airways of cystic fibrosis patients showed that $S$. aureus, $P$. aeruginosa, Rothia mucilaginosa, and Prevotella melaninogenica are the most abundant species [41]. By comparing the core airway microbiomes, the pattern of microbial composition in SARS-CoV-2 infected environment is distinct from those of other diseases. Moreover, there are researches focusing on the physiological environment in the respiratory systems of critically ill COVID-19 patients. Critically ill COVID-19 patient would develop symptoms like ground glass opacities, interstitial thickening, and coarse reticular pattern and so on in the lungs. SARS-CoV-2 virus binds to angiotensin converting enzyme 2 (ACE2) and alters renin-angiotensin system activities, thus leading to enhanced inflammation (increased levels of VEGF, IP-10/CXCL10, MCP-3/ CCL7, IL-6, IL-1, ROS etc.) and development of pulmonary fibrosis [42]. The high neutrophil to lymphocyte ratio leads to the generation of high levels of ROS, which induces host immunopathological response and worsens the conditions of the critically ill COVID-19 patients [43]. In our previous study, we have found that COVID19 patients infected with $P$. aeruginosa express higher levels of IL-6, CRP and PCT [22]. IL6 is a key factor of the cytokine storm of critically ill COVID-19 patients and increased level of IL- 6 associates with higher death rate of the patients $[44,45]$. Studies on cystic fibrosis revealed that the levels of proinflammatory cytokines including IL-1, TNF, IL-6 and IL-8 are higher in CF patients while level of IL-10 is lower comparing to healthy individuals [46]. However, IL-6 levels in the sputum of patients with advanced CF lung disease was extremely low but normal systemic IL-6 production was found [44]. Another study found that early isolate of $P$. aeruginosa from the respiratory system of $\mathrm{CF}$ patients could induce the expression of IL- $1 \beta$ but minimal expression of TNF, IL- 6 and IL-8; while inversely, chronic isolates could induce higher expression of TNF, IL- 6 and IL- 8 and low IL- $1 \beta$ production [47]. Moreover, measurement of circulating and sputum cytokines from CF patients with chronic $P$. aeruginosa infection revealed that higher levels of 
IL-1 receptor antagonist (IRAP), IL-l $\alpha$, IL-1 $\beta$, IL-6 and TNF- $\alpha$ could be detected from sputum, while very little circulating cytokines were detected [48]. In a mouse model with $P$. aeruginosa VAP, the expression of ICAM and VCAM in the lungs and TNF- $\alpha$, IL- $1 \beta$, and IL-6 levels in BALF of wild type mice increased significantly after being infected, while TNF- $\alpha$ is a key regulator of the lung injury caused by $P$. aeruginosa VAP [49]. Thus, comparing with other diseases like CF and VAP, COVID-19 patients retain a distinctive physiological environment in the respiratory system. Considering this together with the results of the present study, SARS-CoV-2 infections can create a specific lung environment that allows rapid evolution and selection of $P$. aeruginosa variants with chronic adapted phenotypes.

\section{Conclusions}

In summary, we demonstrated that $P$. aeruginosa is able to colonize and evolve in SARS-CoV-2 infected environment by altering its alginate biosynthesis and T6SS gene expression, which might enhance biofilm formation, increase antimicrobial resistance and reduce host immune attack during the COVID-19 patients. Our study suggests that post SARS-CoV-2 bacterial superinfection allows the development of bacterial chronic colonizers, which can potentially spread in patients with chronic lung diseases.

\section{Methods}

\section{Isolate collection, bacterial strains and growth media}

Four isolates of $P$. aeruginosa were collected at two different time points longitudinally from sputum samples or BALF of two critically ill COVID-19 patients respectively during routine clinical tests. Isolates collected from patient 1 were named as LYSZa2 and LYSZa3 while isolates collected from patient 2 were named as LYSZa5 and LYSZa6. Luria-Bertani (LB) broth and ABTGC medium supplemented with $10 \%$ Tryptic Soy Broth (TSB) were used for growing cultures. ABTGC medium consists of $0.1 \% \mathrm{MgCl}_{2}, 0.1 \% \mathrm{CaCl}_{2}, 0.1 \% \mathrm{FeCl}_{3}, 0.2 \%$ glucose, $0.2 \%$ casamino acids and $10 \%$ A10 medium which is made of $15.1 \mathrm{mM}\left(\mathrm{NH}_{4}\right)_{2} \mathrm{SO}_{4}, 33.7 \mathrm{mM} \mathrm{Na} \mathrm{HPO}_{4} \cdot 2 \mathrm{H}_{2} \mathrm{O}, 22 \mathrm{mM}$ $\mathrm{KH}_{2} \mathrm{PO}_{4}$ and $0.05 \mathrm{mM} \mathrm{NaCl}$.

\section{Antimicrobial susceptibility tests}

Antimicrobial susceptibility of the isolates to various antibiotics, including ceftazidime, piperacillin, cefoperazone/sulbactam, imipenem, aztreonam, and levofloxacin, were performed using the Kirby-Bauer disc-diffusion method. Diameters of inhibition zone was measured using a Vernier caliper. Susceptibility was determined according to CLSI 2019 [50]. Results of antibiotic susceptibility tests were listed in Additional file 3: Table S1.

\section{Biofilm formation assay}

The isolates were cultured in LB broth at $37^{\circ} \mathrm{C}$ for overnight. The overnight cultures were diluted to $\mathrm{OD}_{600 \mathrm{~nm}}$ 0.01 in fresh LB broth. $100 \mu \mathrm{L}$ of the diluted cultures were loaded into 96-well plate in triplicates and incubated for $24 \mathrm{~h}$ at $37{ }^{\circ} \mathrm{C}$ statically allowing the formation of biofilm. After removing spent media, biofilms were washed carefully with $\mathrm{dd}_{2} \mathrm{O}$ for two times. Biofilms were then stained by $125 \mu \mathrm{L}$ of $0.1 \%$ crystal violet $(\mathrm{CV})$ with $15 \mathrm{~min}$ incubation at room temperature. CV stain in the wells was discarded while stained biofilms were washed twice thoroughly with $\mathrm{ddH}_{2} \mathrm{O}$ and air-dried. Biofilms were then dissolved into $125 \mu \mathrm{L}$ of $30 \%$ acetic acid and quantified relatively by measuring $\mathrm{OD}_{550 \mathrm{~nm}}$ values on a Tecan infinity pro200 microplate reader.

\section{Genome extraction and sequencing}

The isolates were cultured in LB broth at $37{ }^{\circ} \mathrm{C}$ to early stationary phase. For Illumina sequencing, genomic DNA of each isolates was extracted using AxyPerp Bacterial Genomic DNA Miniprep Kit (Corning, New York, USA) following manufacturer's protocol. PCR-free libraries were constructed using VAHTSTM PCR-Free DNA Library Prep Kit for Illumina ${ }^{\circledR}$ (Vazyme, China) following standard protocol. VAHTSTM DNA Adapters for Illumina ${ }^{\circledR}$ (Vazyme, China) was used to tag adaptor to purified fragments. Quality of the libraries were assessed by Agilent Technologies 2100 Bioanalyzer and qPCR. Paired-end DNA sequencing was then performed on Illumina HiSeq X platform with read length of $150 \mathrm{bp}$. For PacBio sequencing, genomes of LYSZa2 and LYSZa5 were extracted using Mabio Bacterial DNA Extraction Mini Kits (Mabio) according to manufacturer's protocol. DNA fragmentation was done using G-tubes (Covaris). SMRTbell DNA template libraries were prepared according to the manufacturer's specification (PacBio, Menlo Park, USA). DNA sequencing was then performed on Pacific Biosciences RSII sequencer (PacBio, Menlo Park, USA).

\section{Transcriptome extraction and sequencing}

The isolates were cultured in triplicates in LB broth at $37{ }^{\circ} \mathrm{C}$ to early stationary phase. Magen HiPure Universal RNA Mini kits (MCBio, China) was used to extract total RNA following the manufacturer's protocol. Extracted RNA was quantified using Qubit 2.0 (Thermo Fisher Scientific, MA, USA) and Nanodrop One (Thermo Fisher Scientific, MA, USA). Quality of RNA samples was assessed by Agilent 2100 system (Agilent Technologies, 
Waldbron, Germany). RNA libraries were constructed according to standard protocol using NEB Next ${ }^{\circledR}$ Ultra $^{\text {TM }}$ Directional RNA Library Prep Kit for Illumina ${ }^{\circledR}$ (New England Biolabs, MA, USA). Ribosomal RNA depletion was carried out using Ribo-zero rRNA Removal Kit. cDNA was synthesized using NEB Next First Strand Synthesis Reaction Buffer. Paired-end RNA sequencing was performed on Illumina NovaSeq 6000 platform with read length of $150 \mathrm{bp}$.

\section{Sequencing data analysis}

Genomic Illumina sequencing reads were imported to CLC Genomics Workbench 20 (Qiagen) and preprocessed using Adaptor trimming function to remove failed reads and adaptor sequences. Clean reads were then assembled into contigs using De Novo Assembly module of CLC Genomics Workbench 20 with default parameters. Single nucleotide polymorphism was detected using 'Resequencing' module of CLC Genomics Workbench 20 based on frequency of more than $80 \%$ using P. aeruginosa PAO1 genome (NC_002516.1) as reference. PacBio sequencing reads were assembled into draft genome using HGAP4 pipeline of SMRT Link software v9.0 with default settings. Rearrangements of draft genomes were checked using Mauve software v.2.4.0 by PROGRESSIVEMAUVE alignment mode [51]. Multilocus sequence typing (MLST) was performed using MLST service available on the Center of Genomic of Epidermiology (CGE) webserver [52]. Identification of antimicrobial resistance genes was performed using ResFinder service on CGE webserver based on $85 \%$ of identity and $60 \%$ of minimal length [53]. Phylogenetic tree was constructed using Parsnp under libMUSCLE alignment mode using draft genomes of the isolates and other strains to construct the core genome tree [54]. Genomic islands on LYSZa2 and LYSZa5 genomes were predicted using IslandViewer 4 webservice using $P$. aeruginosa as reference strain [55]. Circular plot was constructed using BLAST Ring Image Generator using the genomes of $P$. aeruginosa isolates and other $P$. aeruginosa strains downloaded from NCBI database [56]. Genomic loci were visualized using Easyfig package using default setting [57]. Gene names and locus tags labelled on the genomic loci were obtained from Pseudomonas Genome Database [58]. Prediction of protein functions of the genes was performed using NCBI Prokaryotic Genome Annotation Pipeline (PGAP) [59].

RNA sequences were pre-processed, mapped to $P$. aeruginosa PAO1 reference genome and analyzed using RNA analysis module of CLC Genomics Workbench 20 (Qiagen) with default parameters. Total read counts of each sample were normalized and compared using DESeq2 R package. Differentially expressed genes (DEGs) were selected based on absolute fold change $>4$, adjusted p-value $<0.05$ and base mean $>20$ [60]. GO enrichment analysis of DEGs was performed on DAVID bioinformatics database v6.8 [61]. PCoA plot and heatmap were drawn using Vegan, ggplot2, and pheatmap packages in R 4.0.0.

\section{Bacterial competition assay}

Competition between P. aeruginosa and Escherichia coli was carried out following the steps described previously by Hachani et al. [62]. Briefly, the isolates were cultured overnight at $37^{\circ} \mathrm{C}$ on LB agar plates. E. coli/pLacZ strain was grown on LB agar plates containing $40 \mathrm{mg} / \mathrm{mL}$ of 5 -bromo-4-chloro-indolyl- $\beta$-D-galactopyranoside (X-gal) at $37^{\circ} \mathrm{C}$ for overnight and appeared blue in colour. Single colony of each strain was subcultured in TSB medium and grown under agitation for overnight. Appropriate volume of each overnight culture was taken to make final OD600nm $=1$ and centrifuged to collect cells. Cell pellets were resuspended in $100 \mu \mathrm{L}$ TSB medium while $10 \mu \mathrm{L}$ of each suspension was spotted and incubated on LB agar plate for $5 \mathrm{~h}$ at $37^{\circ} \mathrm{C} .30 \mu \mathrm{L}$ of cell suspension of each $P$. aeruginosa strain was taken and mixed gently with 30 $\mu \mathrm{L}$ of $E$. coli cell suspensions. $20 \mu \mathrm{L}$ of mixed cell cultures were spotted onto LB agar plate and incubated for $5 \mathrm{~h}$ at $37{ }^{\circ} \mathrm{C}$. Each bacterial spot was scraped from LB agar plates and resuspended in $1 \mathrm{~mL}$ of TSB medium. Resuspensions of bacterial spots were diluted to $10^{-3}$ serially in tenfold dilutions. These serial cell dilutions were then spotted in triplicates onto LB agar plates containing $40 \mathrm{mg} / \mathrm{mL} \mathrm{X-gal} \mathrm{and} \mathrm{incubated} \mathrm{for} 8 \mathrm{~h}$ for killing E. coli. $100 \mu \mathrm{L}$ of $10^{-3}$ dilution of each mixed bacterial spot were plated onto LB agar plates containing $40 \mathrm{mg} / \mathrm{mL} \mathrm{X-gal} \mathrm{for}$ CFU counting of $E$. coli for quantitating killing effect.

\section{Cytotoxicity assay}

The cytotoxicity of the isolates was assayed by using murine RAW 264.7 macrophages. RAW macrophages were grown in 24-well plates in Dulbecco modified Eagle medium (DMEM), GlutaMAX, sodium pyruvate, and phenol red supplemented with 10\% FBS. Prior to infection, confluent RAW cells were washed twice with sterile PBS and incubated in DMEM medium. Logphase cultures were washed with sterile PBS twice, and resuspended in DMEM medium devoid of FBS. Macrophage cells were infected with the isolates respectively at a Multiplicity of Infection (MOI) of 20 at $37{ }^{\circ} \mathrm{C}$ in $5 \%$ $\mathrm{CO}_{2}$ incubator. After $3 \mathrm{~h}$ infection, the culture supernatants were collected for detecting lactate dehydrogenase (LDH) activities. The LDH activities were detected by using commercially LDH cytotoxicity kit (YAESEN Bio) according to standard procedure. 


\section{ROS production measurement}

ROS production level in RAW264.7 cells cultured for $4 \mathrm{~h}$ after phagocytosis was detected by Reactive Oxygen Species Assay Kit (Beyotime). Briefly, RAW264.7 cells were infected with the isolates respectively at a Multiplicity of Infection (MOI) of 20 at $37{ }^{\circ} \mathrm{C}$ in $5 \% \mathrm{CO}_{2}$ incubator. After $3 \mathrm{~h}$ infection, cells were washed with PBS and incubated in DMEM medium without FBS and DCFH-DA (final concentration $10 \mu \mathrm{M}$ ) reagent at $37^{\circ} \mathrm{C}$ for $20 \mathrm{~min}$, as per the manufacturer's protocols. The cells were washed with PBS and harvested. The stained cells were analysed by using a Cytoflex S flow cytometer (Beckman). All samples were assayed with lasers emitting at $488 \mathrm{~nm}$, and the fluorescence was collected by $530 / 30 \mathrm{~nm}$ bandpass filter. The flow cytometric data were analysed using FlowJo software (BD Biosciences).

\section{Metagenomics analysis}

Nucleic acid of 4 sputum samples (two different time points of two critically ill COVID-19 patients) was extracted and complementary DNA (cDNA) was generated from RNA template by reverse transcription. DNA libraries were constructed through DNA-fragmentation, end-repair, adapter-ligation, and PCR amplification. Qualified libraries were sequenced by BGISEQ-50 platform [63]. High-quality sequencing data were generated by removing low-quality and short (length $<35 \mathrm{bp}$ ) reads, followed by removal of human host sequences mapped to the human reference genome (hg19) using Burrows-Wheeler Alignment [64]. The detailed information of metagenomics data was displayed in Additional file 11: Table S9. The remaining clean data were assembled using the MEGAHIT (version 1.2.9) with default parameters. The open reading frames (ORFs) prediction was then conducted for assembled contigs using Prokka (version 1.12). CD-HIT (version 1.12) was used to cluster genes from each sample based on the parameters (identity $>95 \%$, coverage $>90 \%$ ). We aligned high-quality reads against the gene catalog using Salmon v1.2.1 (identity cutoff $\geq 95 \%$ ) and calculated the corresponding relative abundance of each gene. The taxonomic composition were classified by Kraken 2 [65] by aligning to three Microbial Genome Databases, consisting of 14,459 viruses, 62,319 bacteria, and 1587 fungi. Averages and standard deviations were computed using the base function in R 3.6.2. Venn diagrams were drawn with the Venn Diagram package, while heatmaps were generated using the pheatmap package by R 3.6.2. The $\alpha$-diversity based on Shannon index on the species in each sample was calculated to evaluate the species diversity by $\mathrm{R}$ 3.6.2. Principal Coordinates Analysis (PCoA) was plotted based on Bray-Curtis dissimilarity to compare the species composition of the samples using Vegan package on R 3.6.2.

\section{RT-PCR}

RNA was extracted from the four $P$. aeruginosa isolates using Qiagen RNeasy Mini Kit according to the manufacturer's protocol. After measuring RNA concentration, RNA was quickly converted to cDNA through reverse transcription following the standard steps of HiScript III All-in-one RT SuperMix Perfect for qPCR (Vazyme). RT-PCR was performed using a PowerUpTM SYBRTM Green Master Mix (Thermofisher) according to the manufacturer's protocol. Primers and probes were designed to target $P$. aeruginosa (GCA_000006765.1 ASM676v1). Primers used here are listed in Additional file 12: Table S10. Samples of total DNA from $P$. aeruginosa were diluted to $10 \mathrm{ng} / \mu \mathrm{L} .10$ $\mu \mathrm{L}$ of the total PCR volume was used according to the manufacturer's protocol. The following PCR protocol was used: one cycle at $50{ }^{\circ} \mathrm{C}$ for $2 \mathrm{~min}$ and $95{ }^{\circ} \mathrm{C}$ for 2 min, followed by 45 cycles at $95{ }^{\circ} \mathrm{C}$ for $15 \mathrm{~s}, 57^{\circ} \mathrm{C}$ for $15 \mathrm{~s}$ and $72{ }^{\circ} \mathrm{C}$ for $60 \mathrm{~s}$. LightCycler 96 Software (Roche) was used for data analysis.

\section{Supplementary Information}

The online version contains supplementary material available at https://doi. org/10.1186/s13578-022-00748-z.

Additional file 1: Figure S1. Metagenomic analysis of the sputum of the two patients in which P. aeruginosa isolates LYSZa2, LYSZa3, LYSZa5, and LYSZa6 were sampled indicated that there is no change in the microbial diversity. (A) Box plot showing variation in the abundance of top 20 species as determined by read abundance. (B) Stacked bar plot indicating the top 20 species in the four sputum samples as determined by taxonomic analysis. (C) Alpha diversity indicated by Shannon index within the two groups (A: Ancestor samples including LYSZa2 and LYSZa5; P: progeny samples including LYSZa3 and LYSZa6) and the statistics between the groups. The same letter means that there is no significant difference between the groups (Adjust $p>0.1$ ).

Additional file 2: Figure S2. RT-PCR results of T3SS and T6SS genes. (A) RT-PCR results of the expression of selected T3SS and T6SS genes in LYSZa2 and LYSZa3; (B) RT-PCR results of the expression of selected T3SS and T6SS genes in LYSZa5 and LYSZa6. ${ }^{* *}$ : p-value $<0.01$.

Additional file 3: Table S1. In vitro antimicrobial susceptibility tests of the isolates.

Additional file 4: Table S2. Predicted genomic islands on LYSZa2 genome by IslandViewer4 with at least one prediction method.

Additional file 5: Table S3. Predicted genomic islands on LYSZa5 genome by IslandViewer4 with at least one prediction method.

Additional file 6: Table S4. Genomic accession numbers of $P$. aeruginosa strains selected for phylogenetic tree construction.

Additional file 7: Table S5. Single nucleotide polymorphism (SNP) and other genome modifying events identified between the ancestry isolates and the progeny isolates using PAO1 as reference. SNV Single nucleotide variation, MNV Multi-nucleotide variation.

Additional file 8: Table S6. Full list of DEGs in LYSZa3 comparing to LYSZa2 selected based on the criteria of fold change $\geqq 4$, adjusted p-value $<0.05$ and base mean $\geqq 20$.

Additional file 9: Table S7. Full list of DEGs in LYSZa6 comparing to LYSZa5 selected based on the criteria of fold change $\geqq 4$, adjusted p-value $<0.05$ and base mean $\geqq 20$. 
Additional file 10: Table S8. Common DEGs identified in LYSZa3 and LYSZa6. Filtering criteria are fold change $\geqq 4$, adjusted $p$-value $<0.05$ and base mean $\geqq 20$

Additional file 11: Table S9. Detailed information of metagenomics data of the 4 respiratory samples.

Additional file 12: Table S10. Primers used for RT-PCR tests.

\section{Acknowledgements}

Not applicable.

\section{Authors' contributions}

$J \mathrm{Q}$ and $\mathrm{ZC}$ contributed to manuscript preparation and data analysis. $X \mathrm{D}, \mathrm{HZ}$, $\mathrm{HC}, \mathrm{SH}, \mathrm{KY}, \mathrm{ZJ}, \mathrm{HC}, \mathrm{YZ}, \mathrm{YL}, \mathrm{FB}$ and $\mathrm{YL}$ contributed to data collection, design and operation of laboratory experiments. LL and LY contributed to study design, guide and manuscript verification. All authors read and approved the final manuscript.

\section{Funding}

This work was supported by Guangdong Natural Science Foundation for Distinguished Young Scholar [2020B1515020003] to Dr. Liang Yang, Science and Technology Program of Shenzhen [JCYJ20190809144005609], Guangdong Basic and Applied Basic Research Foundation [2020A1515010586] and Guangdong Province Science and Technology Project [2020B1111170014] to Dr. Jiuxin Qu, and Grant from Bill \& Melinda Gates Foundation to Dr. Lei Liu. This work was supported by grants from Shenzhen Innovation Committee of Science and Technology (ZDSYS20200811144002008 to Shenzhen Key Laboratory of Gene Regulation and Systems Biology) and Shenzhen Science and Technology Program KQTD20200909113758004.

\section{Availability of data and materials}

All Illumina sequencing data used in this study could be found from BioProject No. PRJNA706783 and assembled genomes of $P$. aeruginosa LYSZa2 and P. aeruginosa LYSZa5 could be found from BioProject No. PRJNA712958 and PRJNA712961 on NCBI. The BGISEQ sequencing metagenomics data could be found from BioProject No. PRJNA772037.

\section{Declarations}

\section{Ethics approval and consent to participate}

This work does not include any direct participation of the patients and does not contain any identifiable human data. This work is approved by the Ethics Committee of Shenzhen Third People's Hospital, Second Hospital Affiliated to Southern University of Science and Technology [2020-184] and filed with the Ethics Committee of Southern University of Science and Technology [20200069].

\section{Consent for publication}

Not applicable.

\section{Competing interests}

The authors declare that they have no competing interests.

\section{Author details}

${ }^{1}$ Department of Clinical Laboratory, Shenzhen Third People's Hospital, Second Hospital Affiliated to Southern University of Science and Technology, Guangdong Provincial Clinical Research Center for Infectious Diseases (Tuberculosis), National Clinical Research Center for Infectious Diseases, Shenzhen 518000, Guangdong, China. ${ }^{2}$ School of Medicine, Southern University of Science and Technology, Shenzhen 518055, China. ${ }^{3}$ Medical Research Center, Southern University of Science and Technology Hospital, Shenzhen 518055, China. ${ }^{4}$ School of Biological Sciences, Nankai University, Tianjin 300071, China. ${ }^{5}$ Shenzhen Key Laboratory of Pathogen and Immunity, State Key Discipline of Infectious Disease, Shenzhen Third People's Hospital, Second Hospital Affiliated to Southern University of Science and Technology, Shenzhen 518112, China. ${ }^{6}$ Shenzhen Key Laboratory for Gene Regulation and Systems Biology, Southern University of Science and Technology, Shenzhen 518055, China.
Received: 31 October 2021 Accepted: 19 January 2022

Published online: 09 February 2022

\section{References}

1. Veras FP, et al. SARS-CoV-2-triggered neutrophil extracellular traps mediate COVID-19 pathology. J Exp Med. 2020;217(12):e20201129.

2. Mehta P, et al. COVID-19: consider cytokine storm syndromes and immunosuppression. Lancet. 2020;395(10229):1033-4.

3. Hanada S, et al. Respiratory viral infection-induced microbiome alterations and secondary bacterial pneumonia. Front Immunol. 2018;9:2640.

4. Rawson TM, et al. Bacterial and fungal coinfection in individuals with coronavirus: a rapid review to support COVID-19 antimicrobial prescribing. Clin Infect Dis. 2020;71(9):2459-68.

5. Wang L, et al. Coronavirus disease 2019 in elderly patients: Characteristics and prognostic factors based on 4-week follow-up. J Infect. 2020;80(6):639-45

6. Goyal P, Choi JJ, Safford MM. Clinical characteristics of Covid-19 in New York City. N Engl J Med. 2020;382(24):2372-4.

7. Cox MJ, et al. Co-infections: potentially lethal and unexplored in COVID19. Lancet Microbe. 2020;1(1):e11.

8. Sharifipour $E$, et al. Evaluation of bacterial co-infections of the respiratory tract in COVID-19 patients admitted to ICU. BMC Infect Dis. 2020;20(1):646

9. Zhang $\mathrm{G}$, et al. Clinical features and short-term outcomes of 221 patients with COVID-19 in Wuhan, China. J Clin Virol. 2020;127:104364.

10. Lansbury $L$, et al. Co-infections in people with COVID-19: a systematic review and meta-analysis. J Infect. 2020;81(2):266-75.

11. Arentz $M$, et al. Characteristics and outcomes of 21 critically ill patients with COVID-19 in Washington State. JAMA. 2020;323(16):1612-4.

12. Chen $S$, et al. Clinical and etiological analysis of co-infections and secondary infections in COVID-19 patients: an observational study. Clin Respir J. 2021;15(7):815-25.

13. Gellatly SL, Hancock REW. Pseudomonas aeruginosa: new insights into pathogenesis and host defenses. Pathog Dis. 2013;67(3):159-73.

14. Cole SJ, et al. Catheter-associated urinary tract infection by Pseudomonas aeruginosa is mediated by exopolysaccharide-independent biofilms. Infect Immun. 2014;82(5):2048-58.

15. Luyt CE, et al. Ventilator-associated pneumonia in patients with SARSCOV-2-associated acute respiratory distress syndrome requiring ECMO: a retrospective cohort study. Ann Intensive Care. 2020;10(1):158.

16. Wang $\mathrm{K}$, et al. The rapid in vivo evolution of Pseudomonas aeruginosa in ventilator-associated pneumonia patients leads to attenuated virulence. Open Biol. 2017;7(9):170029.

17. Marvig RL, et al. Convergent evolution and adaptation of Pseudomonas aeruginosa within patients with cystic fibrosis. Nat Genet. 2015;47(1):57.

18. Smith $\mathrm{EE}$, et al. Genetic adaptation by Pseudomonas aeruginosa to the airways of cystic fibrosis patients. Proc Natl Acad Sci USA 2006;103(22):8487-92.

19. Yang $L$, et al. Evolutionary dynamics of bacteria in a human host environment. Proc Natl Acad Sci USA. 2011;108(18):7481-6.

20. Wood TE, et al. The Pseudomonas aeruginosa T6SS delivers a periplasmic toxin that disrupts bacterial cell morphology. Cell Rep. 2019;29(1):187.

21. Gysin M, et al. Antimicrobial susceptibility patterns of respiratory Gramnegative bacterial isolates from COVID-19 patients in Switzerland. Ann Clin Microbiol Antimicrob. 2021;20(1):64

22. Qu J, et al. Persistent bacterial coinfection of a COVID-19 patient caused by a genetically adapted Pseudomonas aeruginosa chronic colonizer. Front Cell Infect Microbiol. 2021;11:641920.

23. Hentzer $M$, et al. Alginate overproduction affects Pseudomonas aeruginosa biofilm structure and function. J Bacteriol. 2001;183(18):5395-401.

24. Imlay JA. Cellular defenses against superoxide and hydrogen peroxide. Annu Rev Biochem. 2008;77:755-76.

25. Qiu D, et al. Regulated proteolysis controls mucoid conversion in Pseudomonas aeruginosa. Proc Natl Acad Sci USA. 2007;104(19):8107-12.

26. Pedersen SS, et al. Pseudomonas aeruginosa alginate in cystic fibrosis sputum and the inflammatory response. Infect Immun. 1990;58(10):3363-8.

27. Limoli DH, et al. Pseudomonas aeruginosa alginate overproduction promotes coexistence with Staphylococcus aureus in a model of cystic fibrosis respiratory infection. MBio. 2017;8(2):e00186. 
28. Chattoraj SS, et al. Pseudomonas aeruginosa alginate promotes Burkholderia cenocepacia persistence in cystic fibrosis transmembrane conductance regulator knockout mice. Infect Immun. 2010;78(3):984-93.

29. Limoli DH, Jones CJ, Wozniak DJ. Bacterial extracellular polysaccharides in biofilm formation and function. Microbiol Spectr. 2015;3(3):3.

30. Malhotra S, Hayes D Jr, Wozniak DJ. Cystic fibrosis and Pseudomonas aeruginosa: the host-microbe interface. Clin Microbiol Rev. 2019;32(3):e00138.

31. Mougous JD, et al. A virulence locus of Pseudomonas aeruginosa encodes a protein secretion apparatus. Science. 2006;312(5779):1526-30.

32. Allsopp LP, et al. RsmA and AmrZ orchestrate the assembly of all three type VI secretion systems in Pseudomonas aeruginosa. Proc Natl Acad Sci USA. 2017:114(29):7707-12

33. Hachani A, Wood TE, Filloux A. Type VI secretion and anti-host effectors. Curr Opin Microbiol. 2016;29:81-93.

34. Ho BT, Dong TG, Mekalanos JJ. A view to a kill: the bacterial type VI secretion system. Cell Host Microbe. 2014;15(1):9-21.

35. Moscoso JA, et al. The Pseudomonas aeruginosa sensor RetS switches type III and type VI secretion via c-di-GMP signalling. Environ Microbiol. 2011;13(12):3128-38.

36. Kordes A, et al. Genetically diverse Pseudomonas aeruginosa populations display similar transcriptomic profiles in a cystic fibrosis explanted lung. Nat Commun. 2019;10(1):3397.

37. Perault Al, et al. Host adaptation predisposes Pseudomonas aeruginosa to type vi secretion system-mediated predation by the Burkholderia cepacia complex. Cell Host Microbe. 2020;28(4):534-47.

38. Rossi E, et al. Pseudomonas aeruginosa adaptation and evolution in patients with cystic fibrosis. Nat Rev Microbiol. 2021;19(5):331-42.

39. Maes $M$, et al. Ventilator-associated pneumonia in critically ill patients with COVID-19. Crit Care. 2021;25(1):25.

40. Gupta S, et al. Comparative analysis of the alveolar microbiome in COPD, ECOPD, Sarcoidosis, and ILD patients to identify respiratory illnesses specific microbial signatures. Sci Rep. 2021;11(1):3963.

41. Bacci $G$, et al. Untargeted metagenomic investigation of the airway microbiome of cystic fibrosis patients with moderate-severe lung disease. Microorganisms. 2020;8(7):1003.

42. Wigen J, et al. Converging pathways in pulmonary fibrosis and Covid19-the fibrotic link to disease severity. Respir Med X. 2020;2:100023.

43. Laforge $M$, et al. Tissue damage from neutrophil-induced oxidative stress in COVID-19. Nat Rev Immunol. 2020;20(9):515-6.

44. Mason K, et al. COVID-19: pathophysiology and implications for cystic fibrosis, diabetes and cystic fibrosis-related diabetes. J Clin Transl Endocrinol. 2021;26:100268.

45. Shekhawat J, et al. Interleukin-6 perpetrator of the COVID-19 cytokine storm. Indian J Clin Biochem. 2021;36(4):1-11.

46. Bonfield TL, et al. Inflammatory cytokines in cystic fibrosis lungs. Am J Respir Crit Care Med. 1995;152(6 Pt 1):2111-8.

47. Phuong MS, et al. Impairment in inflammasome signaling by the chronic Pseudomonas aeruginosa isolates from cystic fibrosis patients results in an increase in inflammatory response. Cell Death Dis. 2021;12(3):241.

48. Kronborg $\mathrm{G}$, et al. Cytokines in sputum and serum from patients with cystic fibrosis and chronic Pseudomonas aeruginosa infection as markers of destructive inflammation in the lungs. Pediatr Pulmonol. 1993;15(5):292-7.

49. Yang YW, et al. Pseudomonas aeruginosa ventilator-associated pneumonia induces lung injury through TNF-a/c-Jun NH2-terminal kinase pathways. PLoS ONE. 2017;12(1):e0169267.

50. CLSI, Performance Standards for Antimicrobial Susceptibility Testing. 29th ed. CLSI supplement M100. Wayne: Clinical and Laboratory Standards Institute, 2019.

51. Darling AE, Mau B, Perna NT. progressiveMauve: multiple genome alignment with gene gain, loss and rearrangement. PLOS ONE. 2010;5(6):e11147.

52. Larsen MV et al. Multilocus sequence typing of total-genome-sequenced bacteria. J Clin Microbiol. 2012;50(4):1355-61.

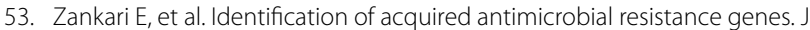
Antimicrob Chemother. 2012;67(11):2640-4

54. Treangen $T$, et al. The harvest suite for rapid core-genome alignment and visualization of thousands of intraspecific microbial genomes. Genome Biol. 2014;15(11):524.
55. Bertelli $C$, et al. IslandViewer 4: expanded prediction of genomic islands for larger-scale datasets. Nucleic Acids Res. 2017;45(W1):W30-5.

56. Alikhan NF, et al. BLAST Ring Image Generator (BRIG): simple prokaryote genome comparisons. BMC Genomics. 2011;12:402.

57. Sullivan MJ, Petty NK, Beatson SA. Easyfig: a genome comparison visualizer. Bioinformatics. 2011;27(7):1009-10.

58. Winsor GL, et al. Enhanced annotations and features for comparing thousands of Pseudomonas genomes in the Pseudomonas genome database. Nucleic Acids Res. 2016:44(D1):D646-53.

59. Tatusova T, et al. NCBI prokaryotic genome annotation pipeline. Nucleic Acids Res. 2016;44(14):6614-24.

60. Love MI, Huber W, Anders S. Moderated estimation of fold change and dispersion for RNA-seq data with DESeq2. Genome Biol. 2014;15(12):550.

61. Huang DW, Sherman BT, Lempicki RA. Systematic and integrative analysis of large gene lists using DAVID bioinformatics resources. Nat Protoc. 2009:4(1):44-57.

62. Hachani A, Lossi NS, Filloux A. A visual assay to monitor T6SS-mediated bacterial competition. J Vis Exp. 2013;73:e50103.

63. Jeon $Y$ J, et al. The feasibility study of non-invasive fetal trisomy 18 and 21 detection with semiconductor sequencing platform. PLoS ONE. 2014;9(10):e110240

64. Li H, Durbin R. Fast and accurate short read alignment with BurrowsWheeler transform. Bioinformatics. 2009;25(14):1754-60.

65. Wood DE, Lu J, Langmead B. Improved metagenomic analysis with Kraken 2. Genome Biol. 2019:20(1):257.

\section{Publisher's Note}

Springer Nature remains neutral with regard to jurisdictional claims in published maps and institutional affiliations.

Ready to submit your research? Choose BMC and benefit from

- fast, convenient online submission

- thorough peer review by experienced researchers in your field

- rapid publication on acceptance

- support for research data, including large and complex data types

- gold Open Access which fosters wider collaboration and increased citations

- maximum visibility for your research: over 100M website views per year

At BMC, research is always in progress.

Learn more biomedcentral.com/submissions 